\title{
Food consumption, physical activity and socio-economic status related to BMI, waist circumference and waist-to-height ratio in adolescents
}

\author{
Sandra Abreu ${ }^{1, *}$, Rute Santos ${ }^{1,2}$, Carla Moreira ${ }^{1}$, Paula Clara Santos ${ }^{1,3}$, Jorge Mota ${ }^{1}$ \\ and Pedro Moreira ${ }^{1,4,5}$ \\ ${ }^{1}$ Research Centre in Physical Activity, Health and Leisure, Faculty of Sport, University of Porto, Rua Dr. Plácido \\ Costa 91, 4200.450 Porto, Portugal: ${ }^{2}$ Maia Institute of Higher Education, Maia, Portugal: ${ }^{3}$ Department of \\ Physical Therapy, School of Health Technology of Porto, Polytechnic Institute of Porto, Vila Nova de Gaia, \\ Portugal: ${ }^{4}$ Faculty of Nutrition and Food Science, University of Porto, Porto, Portugal: ${ }^{5}$ Institute of Public Health, \\ University of Porto, Portugal
}

Submitted 3 September 2012: Final revision received 1 June 2013: Accepted 13 June 2013: First published online 22 July 2013

\begin{abstract}
Objective: To examine the association between obesity and food group intakes, physical activity and socio-economic status in adolescents.

Design: A cross-sectional study was carried out in 2008. Cole's cut-off points were used to categorize BMI. Abdominal obesity was defined by a waist circumference at or above the 90th percentile, as well as a waist-to-height ratio at or above 0.500. Diet was evaluated using an FFQ, and the food group consumption was categorized using sex-specific tertiles of each food group amount. Physical activity was assessed via a self-report questionnaire. Socio-economic status was assessed referring to parental education and employment status. Data were analysed separately for girls and boys and the associations among food consumption, physical activity, socio-economic status and BMI, waist circumference and waist-to-height ratio were evaluated using logistic regression analysis, adjusting the results for potential confounders.

Setting: Public schools in the Azorean Archipelago, Portugal.

Subjects: Adolescents ( $n$ 1209) aged $15-18$ years.

Results: After adjustment, in boys, higher intake of ready-to-eat cereals was a negative predictor while vegetables were a positive predictor of overweight/ obesity and abdominal obesity. Active boys had lower odds of abdominal obesity compared with inactive boys. Boys whose mother showed a low education level had higher odds of abdominal obesity compared with boys whose mother presented a high education level. Concerning girls, higher intake of sweets and pastries was a negative predictor of overweight/obesity and abdominal obesity. Girls in tertile 2 of milk intake had lower odds of abdominal obesity than those in tertile 1. Girls whose father had no relationship with employment displayed higher odds of abdominal obesity compared with girls whose father had high employment status.

Conclusions: We have found that different measures of obesity have distinct associations with food group intakes, physical activity and socio-economic status.
\end{abstract}

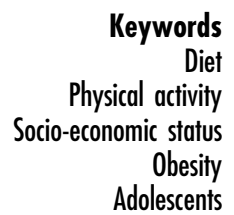

Obesity in children and adolescents is a serious public health problem which is associated with an enhanced risk of chronic diseases such as diabetes, hypertension and cardiovascular failure $^{(1)}$. Furthermore, obesity in childhood increases the likelihood of obesity and its associated com-

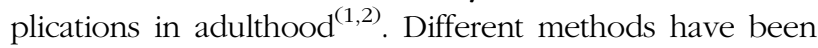
used to identify obesity in childhood and adolescence, the most common being BMI, which is significantly associated with relative fatness in this age group ${ }^{(1)}$. On the other hand, the use of other simple measures to evaluate adiposity, such as waist circumference (WC) or waist-to-height ratio (WHtR), has also been suggested ${ }^{(3,4)}$.

Although obesity is a multifactorial disease, dietary intake and physical activity play an important role in the development of this condition in children and adolescents. Evidence suggests that overweight and obese adolescents often have low physical activity levels and excessive or inadequate consumption of specific food groups ${ }^{(5-7)}$. 
Although the findings are inconsistent, it has been seen that low-nutrient, energy-dense foods (i.e. fast food, sweets and pastries) and beverages (i.e. soda, juice and soft drinks) are associated with enhanced risk of children and adolescents being overweight or obese ${ }^{(8,9)}$. On the other hand, some studies have identified food groups whose intake has an inverse association with obesity, such as fruits, vegetables, ready-to-eat cereals and dairy products ${ }^{(10-12)}$. However, the results regarding the role of specified food groups in the development of overweight/ obesity remain controversial in paediatric populations.

Socio-economic status (SES) has also been associated with childhood obesity. It has been suggested that in developed countries low SES is associated with increased obesity prevalence, whereas in developing countries the opposite seems to be true ${ }^{(13)}$.

In light of the fact that childhood obesity is a public health problem worldwide, there is a critical need to identify related risk factors by evaluating adolescents' food group intakes, physical activity levels and SES, as well as the measures of obesity used with this age group. Thus, the aim of the present study was to examine the associations among the consumption of certain food groups, physical activity, socio-economic factors and different measures of obesity in adolescents.

\section{Materials and methods}

\section{Sampling}

Data for the present cross-sectional study were derived from a school-based study, the Azorean Physical Activity and Health Study II, which aimed to evaluate physical activity, physical fitness, overweight/obesity prevalence, dietary intake, health-related quality of life and other factors in 15- to- 18-year-old adolescents, in 2008. The study was carried out in six of the nine Azorean Islands (São Miguel, Terceira, Faial, Pico, São Jorge and Graciosa), where $95 \%$ of the Azorean population lives ${ }^{(14)}$. The Azorean Archipelago had a population of 246772 habitants in $2011^{(15)}$, holds European ancestry and the main economic activities include services, agriculture and fishing. It is classified as one of the outermost territories of the European Union, and consequently is supported by European Union funds for social and economic development ${ }^{(16)}$. Furthermore, the Azores has some unique social, geographical and urban design features that differ from the mainland. All of the islands are of volcanic origin, hosting numerous landscapes of virgin forest and green fields. Most of the urban areas are small and located on the coast.

All participants in the study were informed of its goals, and the parent or guardian of each participant provided written informed consent for his/her child to participate. The study was approved by the Faculty of Sport, University of Porto and the Portuguese Foundation for Science and
Technology Ethics Committee; it was conducted in accordance with the World Medical Association's Helsinki Declaration for Human Studies.

The population was selected by means of proportionate stratified random sampling, taking into account location (island) and number of students, by age and sex, in each school. The estimated number of students for representativeness of the adolescent population was 1422, but in order to prevent the collection of incomplete information, data were collected for 1515 adolescents. Some adolescents were not included in our analysis ( $n$ 306) as information was missing on their dietary intake ( $n$ 286), BMI or WC ( $n$ 20). Thus, the final sampling resulted in a total of 1209 participants (503 boys). The adolescents who were excluded from the study did not differ significantly from those who were included regarding age $(16 \cdot 2(\mathrm{SD} 1 \cdot 0)$ years $v$. $16 \cdot 1$ (sD $1 \cdot 0)$ years, $P=0 \cdot 158$ ), parental education $(9 \cdot 1$ (sD $4.5)$ years $v \cdot 9 \cdot 1(\mathrm{SD} 4 \cdot 4)$ years, $P=0.890)$ and gender (girls: $61 \cdot 1 \% v \cdot 58 \cdot 4 \%$, boys: $38 \cdot 9 \% v \cdot 41 \cdot 6 \%, P=0 \cdot 388$ ). Finally, the sample was weighted in accordance with the distribution of the Azorean population in schools so as to guarantee the real representativeness of each group (by age and gender).

\section{Antbropometric measures}

Body beight and body weight

Body height and body weight were determined using standard anthropometric methods. Height was measured to the nearest millimetre in bare or stocking feet, with adolescents standing upright against a Holtain portable stadiometer (Crymych, UK). Weight was measured to the nearest $0 \cdot 10 \mathrm{~kg}$, with participants lightly dressed (in underwear and $\mathrm{T}$ shirts), with the use of a portable digital beam scale (Tanita Inner Scan BC 532, Tokyo, Japan).

BMI was calculated using the ratio of weight $/$ height $^{2}$ $\left(\mathrm{kg} / \mathrm{m}^{2}\right)$. Participants were classified as normal weight, overweight or obese, according to age- and sex-specific cut-off points specified by Cole et al. ${ }^{(17,18)}$. Underweight participants $(2.6 \%)$ were combined with participants in the normal weight category, due to the fact that the former represented a small proportion of the overall sample.

\section{Waist circumference and waist-to-height ratio}

For the present study, WC and WHtR were both used as proxy measures of abdominal obesity. WC measurements were taken midway between the tenth rib and the iliac crest and recorded to $0 \cdot 1 \mathrm{~cm}$. A non-elastic flexible tape measure was used, with participants standing erect - arms by sides, feet together and abdomen relaxed - as well as without clothing covering the waist area. Participants were divided into two categories, $<90$ th percentile $(<$ P90) and $\geq 90$ th percentile $(\geq$ P90), according to ageand sex-specific cut-off points specified by Moreno et al. ${ }^{(19)}$. Participants who had $\mathrm{WC} \geq \mathrm{P} 90$ were considered to have abdominal obesity ${ }^{(20)}$. WHtR was calculated as the 
ratio between WC (in centimetres) and height (in centimetres). A WHtR cut-off point of 0.500 was used to define abdominal obesity in males and females ${ }^{(21-23)}$.

\section{Pubertal stage}

To determine pubertal stage (which ranged from 1 to 5), each participant was asked to self-assess his/her stage of development of secondary sexual characteristics. Breast development in girls and genital development in boys was evaluated according to criteria outlined by Tanner and Whitehouse ${ }^{(24)}$.

\section{Sociodemographic and lifestyle variables}

Participants answered a questionnaire that assessed several sociodemographic and lifestyle variables.

\section{Parental education and employment status}

For the present study, mothers' and fathers' education and employment status were both used as proxy measures of SES. Participants were divided into three categories, reflecting divisions within the Portuguese educational system: mandatory or less ( $\leq 9$ school years), secondary (10-12 school years) and college/university ( $>12$ school years). Employment status was divided into four categories, according to the standard Portuguese method of classifying occupations: (i) high employment status (which included armed forces members, representatives of legislative branch offices and agencies, officers, directors and executive officers, and specialists in intellectual and scientific occupations); (ii) medium employment status (which included mid-level technicians and professionals, administrative staff, personal service workers, safety and protective service providers and sellers, and qualified workers in agriculture, fishery and forestry); (iii) low employment status (which included qualified workers in industry and construction, artisans, operators of machinery and equipment, and unqualified workers); and (iv) no relationship with employment (which included pensioners, students and the unemployed) ${ }^{(25,26)}$.

\section{Dietary intake}

Dietary intake was measured via a self-administered semi-quantitative FFQ, validated for the Portuguese population $^{(27)}$. This semi-quantitative FFQ was designed in accordance with criteria laid out by Willett et al. ${ }^{(28)}$ and adapted to include a variety of typical Portuguese food items. The FFQ was adapted for adolescents by including foods more frequently eaten by this age group ${ }^{(29)}$; the adolescent version covered the previous 12 months and comprised ninety-one food items and beverage categories. For each item, the questionnaire offered nine frequency response options, ranging from 'never' to 'six or more times per day', and solicited information on standard portion size and seasonality. Any foods not listed in the questionnaire could be listed by participants in a free-response section. Energy and nutritional intakes were estimated with regard to participants' ratings of the frequency, portion and seasonality of each food and beverage item consumed using the software Food Processor Plus. This program uses nutritional information from the USA that has been adapted for use with typical Portuguese foods and beverages.

For the present study, we defined eleven food groups: (i) dairy (milk, yoghurt and cheese); (ii) milk (whole, semi-skimmed and skimmed); (iii) yoghurt; (iv) cheese; (v) ready-to-eat cereals; (vi) fruits (fresh fruits, including tropical fruits); (vii) vegetables (cabbage, spinach, broccoli, lettuce, peppers, tomatoes, cucumbers, onions, carrots, etc.); (viii) vegetable soup; (ix) sweets and pastries (other biscuits apart from simple ones, croissants, doughnuts, cakes, chocolates, chocolate snacks, quince jam, compote, jelly, honey, sugar, candy); (x) fast food (pizza, hamburgers, mayonnaise, salted snacks); and (xi) sugar-sweetened beverages (soda, juice, fruit juice). Then, participants were categorized using sex-specific tertiles of each food group amount.

\section{Physical activity}

Physical activity was assessed via a self-report questionnaire that evaluated leisure-time physical activities ${ }^{(30)}$. This questionnaire has been shown to have good test-retest reliability among Portuguese adolescents (intra-class correlation coefficient: $0 \cdot 92-0 \cdot 96)^{(31)}$. It consists of five questions with four answer choices (each rated on a 4-point scale): (i) 'Outside school, do you take part in organized sports/ physical activities?'; (ii) 'Outside school, do you take part in non-organized sports/physical activities?'; (iii) 'Outside school hours, how many times a week do you take part in sports or physical activities for at least $20 \mathrm{~min}$ ?; (iv) 'Outside school hours, how many hours a week do you usually take part in physical activities, so much that you get out of breath or sweat?'; and (v) 'Do you take part in competitive sports?' The maximum number of points possible was 20. A physical activity index was obtained for each participant by totalling his/her points, which corresponded to activity level rankings that ranged from 'sedentary' to 'vigorous'. Participants whose physical activity indices were greater than 10 points were classified as 'active', while those whose physical activity indices were 10 points or less were classified as 'low-active ${ }^{,(31,32)}$.

\section{Statistical analysis}

The information concerning boys and girls was analysed separately. The Kolmogorov-Smirnov test was used to verify the variables' normality. The independent-samples $t$ test or the Mann-Whitney test was performed to compare continuous variables between groups, while the $\chi^{2}$ test was used for categorical variables. In this report descriptive analysis is presented in terms of means and standard deviations, unless otherwise stated.

A univariate logistic regression model was used to verify the relationship among overweight/obesity or abdominal 
obesity (WC $\geq \mathrm{P} 90$ or $\mathrm{WHtR} \geq 0 \cdot 500$ ) and each food group consumption, SES and physical activity (Model 1). Variables from the univariate analysis with $P \leq 0.25$ were considered potential independent variables and entered into the logistic regression model as candidate variables for inclusion ${ }^{(33,34)}$. Then we used a conditional stepwise logistic regression model to identify significant variables associated with overweight/obesity or abdominal obesity. A cut-off value of $P<0.05$ was used to include the variables in the multivariate model. The final model was adjusted for age (in years), pubertal stage, energy intake (in $\mathrm{kJ} / \mathrm{kcal}$ ) and dietary fibre (in $\mathrm{g} / 4184 \mathrm{~kJ}$ (1000 kcal); Model 2). Age, energy intake and dietary fibre were entered as continuous variables. Furthermore, we adjusted the final logistical model for under-reporting of energy intake, which was estimated using the ratio between reported energy intake and predicted BMR (EI:BMR) ${ }^{(35-37)}$. The thresholds that defined low-energy reporters (underreporters) were EI:BMR $\leq 1.70$ and $\leq 1.71$ for girls and boys between 15 and 17 years old and EI:BMR $\leq 1.67$ and $\leq 1.81$ for girls and boys aged 18 years. 'Low-energy reporter' (a categorical variable) was included in the final model as a confounding factor.

Odds ratios and $95 \%$ confidence intervals were computed for overweight/obesity and abdominal obesity (WC $\geq$ P90 or $\mathrm{WHtR} \geq 0 \cdot 500$ ), according to food group intakes, physical activity and SES. A $P$ value of $<0.05$ was regarded as significant. All analyses were performed using the statistical software package IBM SPSS Statistics Version 20.

\section{Results}

Descriptive characteristics of the adolescents in the sample, according to their BMI, WC and WHtR status, are shown in Tables 1 and 2. Within both genders, adolescents who were classified as overweight/obese or abdominally obese presented higher weight, BMI, WC and WHtR values $(P<0 \cdot 001$, for all). Girls with abdominal obesity had a higher proportion of fathers with low education level and were more likely to have two parents with low employment status $(P<0 \cdot 05$, for all). Concerning physical activity, boys with abdominal obesity were less active than their lean counterparts $(P<0 \cdot 05$, for all). Concerning girls, no significant differences were seen in physical activity between the groups, regardless of their BMI, WC or WHtR status. Our data showed that $16 \cdot 7 \%$ (boys: $17 \cdot 1 \% v$. girls: 19.3\%) of adolescents reported being in Tanner stage 3 or lower, while $59 \cdot 1 \%$ reported being in stage 4 (boys: $59 \cdot 0 \% v$. girls: $59 \cdot 1 \%$ ) and $24 \cdot 2 \%$ in stage 5 (boys: $21 \cdot 7 \%$ $v$. girls: $27 \cdot 8 \%)$.

The adolescents' nutritional and dietary characteristics, and their food group consumption, according to BMI, WC and WHtR status, are presented in Tables 3 and 4 . In both genders, adolescents who were overweight/obese or abdominally obese had lower intakes of ready-to-eat cereals, sweets and pastries $(P<0 \cdot 05$, for all). Overweight/ obese or abdominally obese boys had also lower intakes of vegetables $(P<0 \cdot 05$, for all). Girls who were overweight/ obese or abdominally obese had lower energy and sugarsweetened beverage intakes $(P<0 \cdot 05$, for all). Lower dairy and milk consumption was seen in girls with abdominal obesity, compared with those classified as without abdominal obesity $(P<0 \cdot 05$, for all). In both genders there was no significant difference across groups concerning yoghurt and fruit intakes.

Univariate associations of overweight/obesity and abdominal obesity ( $\mathrm{WC} \geq \mathrm{P} 90$ or $\mathrm{WHtR} \geq 0 \cdot 500$ ), according to consumption of the eleven food groups measured, physical activity and SES, are shown in Table 5 . The variables that remained in the model after the conditional stepwise method are presented in Table 6. After adjustment, in boys, higher intake of ready-to-eat cereals was a negative predictor and higher intake of vegetables was a positive predictor of overweight/obesity and abdominal obesity. Active boys had lower odds of abdominal obesity (WHtR $\geq 0 \cdot 500$ ) compared with inactive boys (OR $=0.454 ; 95 \% \mathrm{CI} 0 \cdot 234$, $0 \cdot 880$ ). Boys whose mother had a low education level had higher odds of abdominal obesity (WHtR $\geq 0 \cdot 500$ ) compared with boys whose mother presented a high education level (secondary: $\mathrm{OR}=3 \cdot 054 ; 95 \%$ CI $1 \cdot 085$, 8.596; mandatory or less: $\mathrm{OR}=3 \cdot 172 ; 95 \%$ CI $1 \cdot 200$, 8.382). In girls, higher intake of sweets and pastries was a negative predictor of overweight/obesity and abdominal obesity. Girls in tertile 2 of milk intake had lower odds of abdominal obesity (WC $\geq \mathrm{P} 90$ and $\mathrm{WHtR} \geq 0 \cdot 500$ ) than those in tertile 1. Girls whose father had no relationship with employment had higher odds of abdominal obesity (defined by WC $\geq$ P90) compared with girls whose father presented a high employment status $(\mathrm{OR}=2 \cdot 672 ; 95 \%$ CI $1 \cdot 062,6 \cdot 726)$.

\section{Discussion}

The present study explored the relationship among food group intakes, physical activity and SES and obesity, as well as different measures of abdominal obesity (i.e. WC and WHtR). After adjustments the results suggested that the intake of ready-to-eat cereals in boys, and intakes of milk and sweets and pastries in girls, were negative predictors of overweight/obesity or abdominal obesity. It was also seen that higher vegetable intake was a positive predictor of overweight/obesity and abdominal obesity only in boys. On the other hand, physical activity seems to be negatively associated with abdominal obesity in boys. In addition, mother's education level in boys and father's employment status in girls were positive predictors of abdominal obesity.

Few studies have examined the relationship between specific types of dairy intake and measures of abdominal 
Table 1 Characteristics of the boys' sample, according to BMI, waist circumference and waist-to-height ratio status: adolescent boys aged 15-18 years, Azorean Archipelago, Portugal, 2008

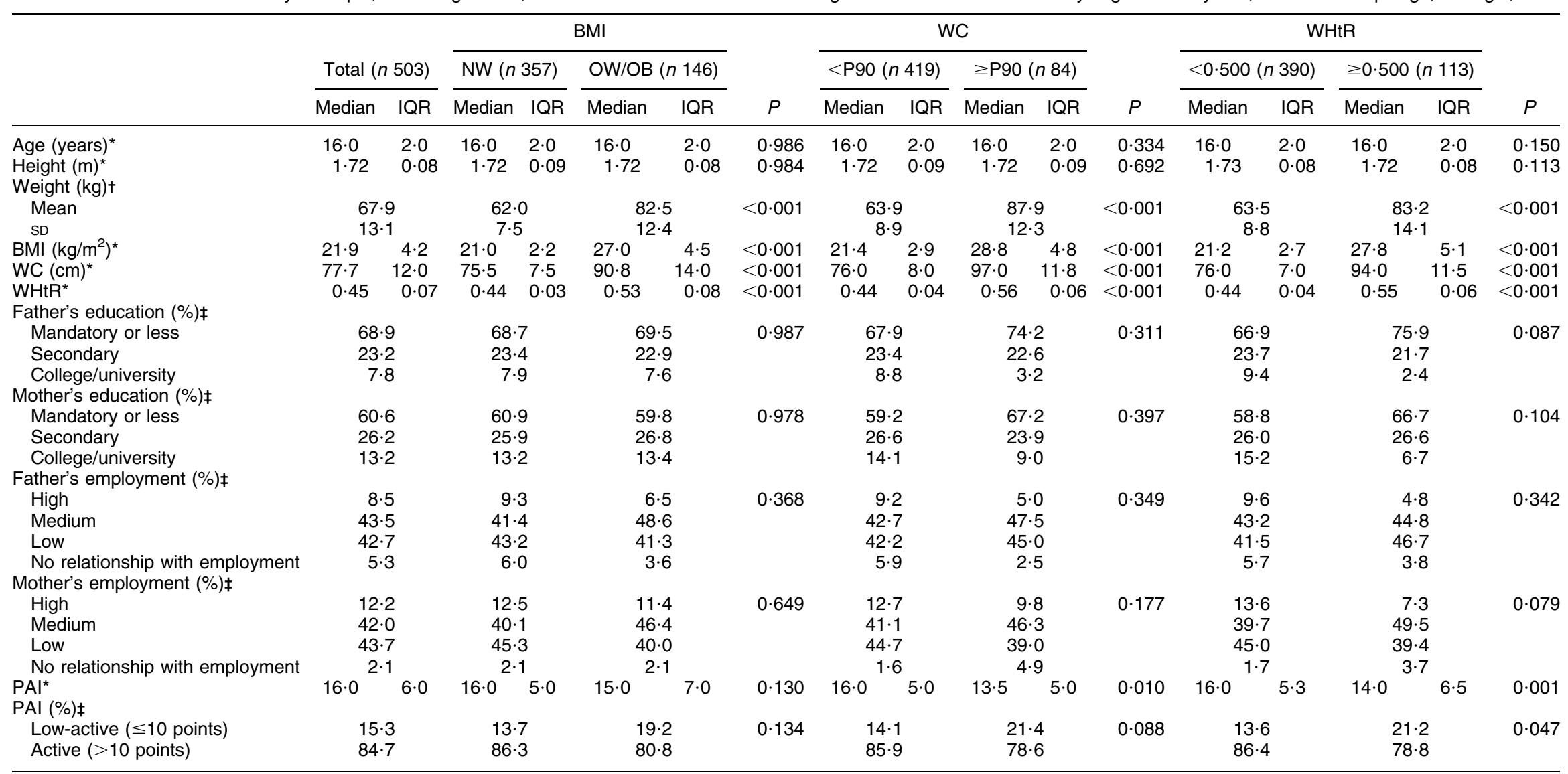

WC, waist circumference; WHtR, waist-to-height ratio; NW, normal weight; OW/OB, overweight and obese; P90, 90th percentile; IQR, interquartile range; PAI, physical activity index. *Analysis by Mann-Whitney's test for continuous variables.

tAnalysis by $t$ test for continuous variables. 
Table 2 Characteristics of the girls' sample, according to BMI, waist circumference and waist-to-height ratio status: adolescent girls aged 15-18 years, Azorean Archipelago, Portugal, 2008

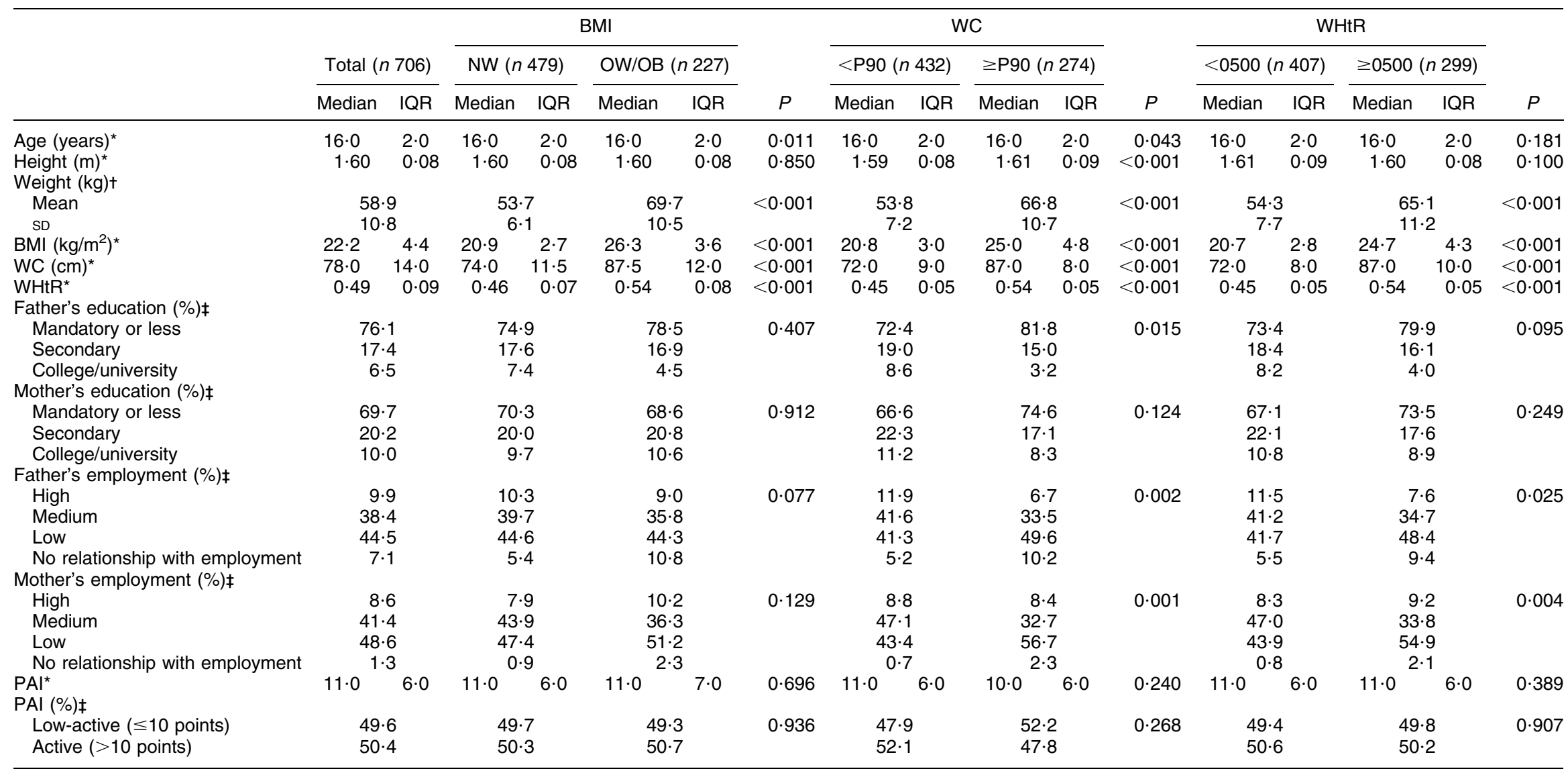

WC, waist circumference; WHtR, waist-to-height ratio; NW, normal weight; OW/OB, overweight and obese; P90, 90th percentile; IQR, interquartile range; PAI, physical activity index. *Analysis by Mann-Whitney's test for continuous variables.

Analysis by $t$ test for continuous variables. 
Table 3 Dietary and nutritional characteristics of the boys' sample, according to BMI, waist circumference and waist-to-height ratio status: adolescent boys aged 15-18 years, Azorean Archipelago, Portugal, 2008

\begin{tabular}{|c|c|c|c|c|c|c|c|c|c|c|c|c|c|c|c|c|c|}
\hline & & & \multicolumn{4}{|c|}{ BMI } & \multirow[b]{3}{*}{$P$} & \multicolumn{4}{|c|}{ WC } & \multirow[b]{3}{*}{$P$} & \multicolumn{4}{|c|}{$\mathrm{WHtR}$} & \multirow[b]{3}{*}{$P$} \\
\hline & \multicolumn{2}{|c|}{ Total $(n$ 503) } & \multicolumn{2}{|c|}{ NW ( $n$ 357) } & \multicolumn{2}{|c|}{ OW/OB (n 146) } & & \multicolumn{2}{|c|}{$<\mathrm{P} 90$ (n 419) } & \multicolumn{2}{|c|}{$\geq \mathrm{P} 90(n 84)$} & & \multicolumn{2}{|c|}{$<0.500(n 390)$} & \multicolumn{2}{|c|}{$\geq 0.500(n 113)$} & \\
\hline & Median & IQR & Median & IQR & Median & IQR & & Median & IQR & Median & IQR & & Median & IQR & Median & IQR & \\
\hline Energy intake $(\mathrm{kJ} / \mathrm{d})^{\star}$ & 10427 & 6986 & 10468 & 7193 & 10144 & 6752 & 0.376 & 10468 & 7258 & 9759 & 6202 & 0.205 & 10483 & 7228 & 9917 & 6576 & $0 \cdot 182$ \\
\hline Energy intake $(\mathrm{kcal} / \mathrm{d})^{\star}$ & $2492 \cdot 1$ & $1669 \cdot 8$ & $2501 \cdot 9$ & $1719 \cdot 2$ & $2424 \cdot 4$ & $1613 \cdot 8$ & 0.376 & $2501 \cdot 9$ & $1734 \cdot 6$ & $2332 \cdot 5$ & $1482 \cdot 3$ & 0.205 & $2505 \cdot 6$ & $1727 \cdot 5$ & $2370 \cdot 2$ & $1571 \cdot 7$ & $0 \cdot 182$ \\
\hline Protein $(\%$ of energy) $\dagger$ & \multirow{2}{*}{\multicolumn{2}{|c|}{$\begin{array}{r}17 \cdot 9 \\
3 \cdot 9\end{array}$}} & & & \multirow{2}{*}{\multicolumn{2}{|c|}{$17 \cdot 8$}} & & & & & & & & & & & \\
\hline $\begin{array}{l}\text { Mean } \\
\text { SD }\end{array}$ & & & $18 \cdot 0$ & $\cdot 0$ & & & 0.750 & $\begin{array}{r}17 \\
4\end{array}$ & $\cdot 8$ & & $\cdot 3$ & 0.319 & & & & $\cdot 9$ & 0.819 \\
\hline Carbohydrate (\% of energy)† & & & & & & & & & & & & & & & & & \\
\hline $\begin{array}{l}\text { Mean } \\
\text { SD }\end{array}$ & $\begin{array}{r}48 \\
7\end{array}$ & & $\begin{array}{r}48 \\
7\end{array}$ & $\begin{array}{r}\cdot 1 \\
8\end{array}$ & & $\begin{array}{l}1 \\
0 \\
0\end{array}$ & $0 \cdot 184$ & $\begin{array}{r}48 \\
7\end{array}$ & .4 & & .5 & 0.923 & & & & $\cdot 3$ & $0 \cdot 155$ \\
\hline Total fat (\% of energy) + & & & & & & & & & & & & & & & & & \\
\hline $\begin{array}{l}\text { Mean } \\
\text { SD }\end{array}$ & $\begin{array}{r}32 . \\
5\end{array}$ & $\cdot 8$ & $\begin{array}{r}33 \\
5\end{array}$ & $\cdot 1$ & & $\begin{array}{r}0 \\
\cdot 7\end{array}$ & 0.043 & $\begin{array}{r}32 \\
5\end{array}$ & $\cdot 9$ & & $\cdot 3$ & 0.380 & & & & $\cdot 8$ & 0.030 \\
\hline Dietary fibre $(\mathrm{g} / 4184 \mathrm{~kJ}(1000 \mathrm{kcal}))^{\star}$ & $8 \cdot 8$ & $3 \cdot 8$ & 8.5 & $3 \cdot 7$ & $9 \cdot 7$ & $4 \cdot 2$ & 0.003 & $8 \cdot 8$ & $3 \cdot 8$ & $9 \cdot 1$ & $4 \cdot 2$ & 0.302 & $8 \cdot 7$ & $3 \cdot 8$ & $9 \cdot 4$ & $4 \cdot 0$ & $0 \cdot 126$ \\
\hline Dairy $(g / d)^{*}$ & $528 \cdot 6$ & $506 \cdot 9$ & $551 \cdot 8$ & $507 \cdot 5$ & $428 \cdot 9$ & $504 \cdot 0$ & $0 \cdot 388$ & $528 \cdot 6$ & $506 \cdot 9$ & $534 \cdot 6$ & $485 \cdot 2$ & 0.647 & $557 \cdot 4$ & $503 \cdot 2$ & $400 \cdot 0$ & $515 \cdot 8$ & 0.660 \\
\hline Tertile $1(\%)$ & & & 31 & $\cdot 7$ & & & $0 \cdot 689 \ddagger$ & 32 & 5 & & 5 & $0.926 \ddagger$ & & & & 4 & $0.762 \ddagger$ \\
\hline Tertile $2(\%)$ & & & 33 & $\cdot 1$ & & & & 32 & 5 & & $\cdot 1$ & & & & & $\cdot 1$ & \\
\hline Tertile 3 (\%) & & & 35 & & & & & 35 & $\cdot 1$ & & $\cdot 3$ & & & & & .5 & \\
\hline Milk $(\mathrm{g} / \mathrm{d})^{*}$ & $305 \cdot 0$ & 383.5 & $348 \cdot 6$ & $366 \cdot 0$ & $260 \cdot 3$ & $418 \cdot 3$ & $0 \cdot 487$ & $305 \cdot 0$ & $366 \cdot 0$ & $252 \cdot 7$ & $418 \cdot 3$ & 0.599 & $331 \cdot 4$ & $366 \cdot 0$ & $245 \cdot 0$ & $418 \cdot 3$ & 0.583 \\
\hline Tertile 1 (\%) & & & 46 & & & & $0 \cdot 790 \ddagger$ & 46 & & & $\cdot 8$ & $0 \cdot 911 \ddagger$ & & & & .7 & $0.822 \ddagger$ \\
\hline Tertile $2(\%)$ & & & 32 & & & & & 32 & & & 8 & & & & & $\cdot 2$ & \\
\hline Tertile 3 (\%) & & & 21 & 0 & & & & 21 & $\cdot 5$ & & $\cdot 4$ & & & & & 1 & \\
\hline Yoghurt $(\mathrm{g} / \mathrm{d})^{\star}$ & $53 \cdot 6$ & $116 \cdot 7$ & $53 \cdot 6$ & $89 \cdot 9$ & $35 \cdot 8$ & $116 \cdot 7$ & 0.882 & $53 \cdot 6$ & $89 \cdot 9$ & $17 \cdot 9$ & $116 \cdot 7$ & 0.909 & $53 \cdot 6$ & $89 \cdot 9$ & $53 \cdot 6$ & $116 \cdot 7$ & 0.451 \\
\hline Tertile 1 (\%) & & & 43 & & & & $0 \cdot 237 \ddagger$ & 44 & & & & $0 \cdot 542 \ddagger$ & & & & $\cdot 2$ & $0.974 \ddagger$ \\
\hline Tertile 2 (\%) & & & 22 & & & & & 21 & 0 & & $\cdot 7$ & & & & & $\cdot 4$ & \\
\hline Tertile 3 (\%) & & & 34 & & 34 & & & 34 & $\cdot 8$ & & $\cdot 3$ & & 3 & & 35 & 4 & \\
\hline Cheese $(\mathrm{g} / \mathrm{d})^{*^{*}}$ & $12 \cdot 9$ & $28 \cdot 0$ & $12 \cdot 9$ & $26 \cdot 0$ & $12 \cdot 9$ & $28 \cdot 0$ & 0.418 & $12 \cdot 9$ & $28 \cdot 0$ & $12 \cdot 9$ & $28 \cdot 0$ & 0.920 & $12 \cdot 9$ & $28 \cdot 0$ & $12 \cdot 9$ & $28 \cdot 0$ & 0.683 \\
\hline Tertile 1 (\%) & & & 25 & & & & $0 \cdot 278 \ddagger$ & 26 & & & & $0 \cdot 850 \ddagger$ & & & & $\cdot 2$ & $0 \cdot 824 \ddagger$ \\
\hline Tertile 2 (\%) & & & 34 & & & & & 33 & & & $\cdot 1$ & & & & & 6 & \\
\hline Tertile 3 (\%) & & & 40 & & & & & 39 & & & $\cdot 1$ & & & & & 2 & \\
\hline $\operatorname{RTEC}(\mathrm{g} / \mathrm{d})^{\star}$ & $17 \cdot 1$ & $34 \cdot 3$ & $31 \cdot 4$ & $34 \cdot 3$ & $17 \cdot 1$ & $37 \cdot 3$ & 0.004 & $31 \cdot 4$ & $34 \cdot 3$ & $17 \cdot 1$ & $37 \cdot 3$ & 0.010 & $31 \cdot 4$ & $34 \cdot 3$ & $17 \cdot 1$ & $37 \cdot 3$ & 0.001 \\
\hline Tertile 1 (\%) & & & 28 & & & & $0.001 \ddagger$ & 29 & & & & $0 \cdot 008 \ddagger$ & & & & .5 & $0.001 \ddagger$ \\
\hline Tertile 2 (\%) & & & 56 & & & & & 57 & & & $\cdot 3$ & & & & & 0 & \\
\hline Tertile 3 (\%) & & & 14 & & & 1 & & 13 & 6 & & $\cdot 4$ & & & & & .5 & \\
\hline Fruits $(g / d)^{*}$ & $200 \cdot 1$ & $295 \cdot 9$ & $191 \cdot 5$ & $280 \cdot 1$ & $222 \cdot 4$ & $317 \cdot 0$ & $0 \cdot 136$ & $197 \cdot 6$ & $292 \cdot 4$ & $214 \cdot 0$ & $303 \cdot 2$ & 0.595 & $197 \cdot 6$ & $281 \cdot 0$ & $219 \cdot 2$ & $342 \cdot 3$ & $0 \cdot 268$ \\
\hline Tertile 1 (\%) & & & 35 & & & & $0 \cdot 310 \ddagger$ & 33 & & & & $0 \cdot 694 \ddagger$ & & & & 1 & $0.479 \ddagger$ \\
\hline Tertile $2(\%)$ & & & 33 & & & & & 33 & $\cdot 4$ & & & & & & & .9 & \\
\hline Tertile 3 (\%) & & & 31 & & & & & 32 & $\cdot 7$ & & .9 & & & & & 1 & \\
\hline Vegetables $(\mathrm{g} / \mathrm{d})^{\star}$ & $65 \cdot 7$ & $116 \cdot 6$ & $59 \cdot 8$ & $107 \cdot 1$ & $86 \cdot 9$ & $116 \cdot 8$ & 0.008 & $61 \cdot 9$ & $125 \cdot 4$ & $89 \cdot 5$ & $115 \cdot 1$ & 0.020 & $60 \cdot 7$ & 114.9 & $86 \cdot 9$ & $106 \cdot 9$ & 0.034 \\
\hline Tertile $1(\%)$ & & & 37 & & & & $0.019 \ddagger$ & 35 & & & & $0.020 \ddagger$ & & & & .2 & $0.005 \ddagger$ \\
\hline Tertile 2 (\%) & & & 31 & & & & & 31 & $\cdot 7$ & & $\cdot 7$ & & & & & 4 & \\
\hline Tertile $3(\%)$ & & & 31 & & & & & 32 & & & & & & & & 4 & \\
\hline Vegetable soup $(\mathrm{g} / \mathrm{d})^{*}$ & $42 \cdot 1$ & $106 \cdot 8$ & $42 \cdot 1$ & $106 \cdot 8$ & $42 \cdot 1$ & $212 \cdot 1$ & 0.754 & $42 \cdot 1$ & $106 \cdot 8$ & $42 \cdot 1$ & $106 \cdot 8$ & 0.569 & $42 \cdot 1$ & $106 \cdot 8$ & $42 \cdot 1$ & $106 \cdot 8$ & 0.587 \\
\hline Tertile $1(\%)$ & & & 32 & & & & $0.982 \ddagger$ & 32 & & & & $0 \cdot 779 \ddagger$ & & & & .5 & $0.667 \ddagger$ \\
\hline Tertile $2(\%)$ & & & 21 & & & & & 21 & 0 & & $\cdot 4$ & & & & & 0 & \\
\hline Tertile $3(\%)$ & & & 45 & & & & & 46 & & & & & & & & .5 & \\
\hline Sweets and pastries $(\mathrm{g} / \mathrm{d})^{*}$ & $44 \cdot 4$ & $57 \cdot 8$ & $48 \cdot 5$ & $65 \cdot 2$ & $39 \cdot 5$ & $49 \cdot 2$ & 0.004 & $47 \cdot 6$ & $60 \cdot 6$ & $33 \cdot 4$ & $44 \cdot 2$ & 0.004 & $48 \cdot 2$ & $62 \cdot 7$ & $35 \cdot 6$ & $41 \cdot 0$ & 0.002 \\
\hline Tertile $1(\%)$ & & & 30 & & & & $0.086 \ddagger$ & 31 & & & & $0.041 \ddagger$ & & & & 0 & $0.086 \ddagger$ \\
\hline Tertile 2 (\%) & & & 33 & & & & & 33 & 2 & & & & & & & 2 & \\
\hline Tertile $3(\%)$ & & & 36 & & & & & 35 &.$\overline{6}$ & & .6 & & & & & 7 & \\
\hline
\end{tabular}


obesity in children and adolescents. Data from the Third National Health and Nutrition Examination Survey showed that mean dairy intake was inversely associated with central obesity in adolescents ${ }^{(38)}$. In a cross-sectional study with children aged 10 years, increased milk consumption was also associated with lower WC, whereas no significant association was seen with $\mathrm{BMI}^{(39)}$, as in our study. It has been described that BMI is the most convenient way of indirectly measuring body fat ${ }^{(1)}$. However, the same BMI percentile does not represent the same percentage body fatness at different ages for boys and girls ${ }^{(40)}$, and this may contribute to the specific difference in the findings. In the present study we found a significant inverse association between milk intake and abdominal obesity only in girls. Evidence suggests that gender may influence body composition, with girls having greater body fat ${ }^{(41,42)}$, and it is possible that the interaction between milk (and its components) and body fat may differ across different body fat thresholds ${ }^{(43)}$. In accordance with this, Vergnaud et al. ${ }^{(44)}$ reported that milk and yoghurt intakes were protective against 6-year changes in body weight only in adults who were initially overweight. Furthermore, although in our study no inverse association was found between the higher and lower tertile, higher consumption of milk did not increase the probability of being obese, as other studies had reported ${ }^{(45,46)}$. A number of possible explanations have been suggested for the protective effect of milk intake on obesity. Milk is an important source of $\mathrm{Ca}$, which appears to play a significant role in the regulation of energy metabolism by reducing the levels of lipogenesis in adipocytes and increasing both faecal fat excretion and fat oxidation ${ }^{(47)}$. Moreover, milk proteins, especially whey proteins, have been positively associated with satiety ${ }^{(47)}$. On the other hand, milk compounds may also be involved in body fat distribution. It has described that visceral adipose tissue has greater amounts of 11- $\beta$-hydroxysteroid dehydrogenase type $1^{(48)}$, which is over-expressed in vitro in those with central adiposity ${ }^{(49)}$. It has been suggested that a high-Ca and high-dairy diet down-regulates 11- $\beta$-hydroxysteroid dehydrogenase type 1 expression and decreases the concentration of glucocorticoid, which consequently decreases the size of adipose fat deposits ${ }^{(48)}$.

Concerning ready-to-eat cereal intake, our results are in accordance with evidence suggesting that ready-to-eat cereal consumption protects against childhood obesity. Kafatos et al. ${ }^{(11)}$ found that adolescents who are daily consumers of ready-to-eat cereals had lower mean BMI, WC and WHtR values, compared with non-consumers and occasional consumers. Other cross-sectional ${ }^{(50-52)}$ and prospective studies ${ }^{(53,54)}$ have shown similar results. According to the latter studies, the 'antiobesity' effect of ready-to-eat cereals may be due to the association of these cereals with higher breakfast consumption and milk and dietary fibre intake, which have been associated with lower risk of obesity. 
Table 4 Dietary and nutritional characteristics of the girls' sample, according to BMI, waist circumference and waist-to-height ratio status: adolescent girls aged 15-18 years, Azorean Archipelago, Portugal, 2008

\begin{tabular}{|c|c|c|c|c|c|c|c|c|c|c|c|c|c|c|c|c|c|}
\hline & \multirow{2}{*}{\multicolumn{2}{|c|}{ Total $(n$ 706) }} & \multicolumn{4}{|c|}{ BMI } & \multirow[b]{3}{*}{$P$} & \multicolumn{4}{|c|}{ WC } & \multirow[b]{3}{*}{$P$} & \multicolumn{4}{|c|}{$\mathrm{WHtR}$} & \multirow[b]{3}{*}{$P$} \\
\hline & & & \multicolumn{2}{|c|}{ NW ( $n$ 479) } & \multicolumn{2}{|c|}{ OW/OB (n 227) } & & \multicolumn{2}{|c|}{ <P90 (n 432) } & \multicolumn{2}{|c|}{$\geq \mathrm{P} 90(n 274)$} & & \multicolumn{2}{|c|}{$<0.500(n 407)$} & \multicolumn{2}{|c|}{$\geq 0.500$ (n 299) } & \\
\hline & Median & IQR & Median & IQR & Median & IQR & & Median & IQR & Median & IQR & & Median & IQR & Median & IQR & \\
\hline Energy intake $(\mathrm{kJ} / \mathrm{d})^{\star}$ & 9166 & 5229 & 9629 & 5612 & 8352 & 4651 & & 9629 & 5330 & 8354 & 5233 & 0.004 & 2306 & 5376 & 8353 & 5540 & $<0.001$ \\
\hline Energy intake $(\mathrm{kcal} / \mathrm{d})^{*}$ & $2190 \cdot 7$ & $1249 \cdot 7$ & $2301 \cdot 4$ & $1341 \cdot 3$ & $1996 \cdot 2$ & $1111 \cdot 5$ & $<0.001$ & $2301 \cdot 4$ & $1273 \cdot 9$ & $1996 \cdot 6$ & $1250 \cdot 7$ & 0.004 & $2301 \cdot 7$ & $1284 \cdot 9$ & $1996 \cdot 4$ & $1324 \cdot 0$ & $<0.001$ \\
\hline \multicolumn{18}{|l|}{ Protein (\% of energy)t } \\
\hline Mean & \multirow{2}{*}{\multicolumn{2}{|c|}{$\begin{array}{r}17 \cdot 7 \\
3 \cdot 7\end{array}$}} & \multicolumn{2}{|c|}{$17 \cdot 3$} & & & 0.001 & 17 & 5 & 17 & 9 & 0.219 & 17 & 3 & 18 & & 0.014 \\
\hline SD & & & & 5 & & 0 & & & 4 & & $\cdot 1$ & & & 4 & 4. & $\cdot 1$ & \\
\hline Carbohydrate $(\%$ of energy) + & & & & & & & & & & & & & & & & & \\
\hline $\begin{array}{l}\text { Mean } \\
\text { SD }\end{array}$ & $\begin{array}{r}49 . \\
7 .\end{array}$ & .9 & $\begin{array}{r}50 \\
7\end{array}$ & 3 & & $\begin{array}{l}\cdot 0 \\
.0\end{array}$ & 0.041 & $\begin{array}{r}50 \\
7\end{array}$ & $\cdot 1$ & $\begin{array}{r}49 \\
8\end{array}$ & $\cdot 6$ & 0.432 & $\begin{array}{r}50 \\
7\end{array}$ & $\cdot 4$ & $\begin{array}{r}49 \\
8\end{array}$ & $\cdot 3$ & 0.089 \\
\hline Total fat (\% of energy) $\dagger$ & & & & & & & & & & & & & & & & & \\
\hline Mean & $\begin{array}{r}32 . \\
5 .\end{array}$ & .0 & $\begin{array}{r}31 \\
5\end{array}$ & .9 & & $\begin{array}{r}\cdot 1 \\
.0\end{array}$ & 0.657 & $\begin{array}{r}31 \\
5\end{array}$ & .9 & $\begin{array}{r}32 \\
6\end{array}$ & 1 & 0.758 & $\begin{array}{r}31 \\
5\end{array}$ & .9 & $\begin{array}{r}32 \\
6\end{array}$ & $\cdot 1$ & 0.504 \\
\hline Dietary fibre $(\mathrm{g} / 4184 \mathrm{~kJ}(1000 \mathrm{kcal}))^{*}$ & $9 \cdot 8$ & $4 \cdot 3$ & $9 \cdot 9$ & $4 \cdot 3$ & $9 \cdot 7$ & 4.5 & 0.790 & $9 \cdot 8$ & $4 \cdot 0$ & $9 \cdot 9$ & $4 \cdot 6$ & 0.226 & $9 \cdot 8$ & $4 \cdot 3$ & $9 \cdot 8$ & $4 \cdot 4$ & 0.621 \\
\hline Dairy $(\mathrm{g} / \mathrm{d})^{*}$ & $399 \cdot 0$ & $465 \cdot 6$ & $428 \cdot 0$ & $466 \cdot 0$ & $373 \cdot 3$ & $442 \cdot 7$ & 0.332 & $560 \cdot 1$ & $467 \cdot 6$ & $355 \cdot 1$ & $460 \cdot 8$ & 0.001 & $586 \cdot 5$ & $466 \cdot 6$ & $355 \cdot 1$ & $462 \cdot 8$ & 0.001 \\
\hline Tertile $1(\%)$ & & & 32 & & & $\cdot 8$ & $0.693 \ddagger$ & 29 & $\cdot 2$ & 39 & $\cdot 8$ & $0.006 \ddagger$ & 29 & & 39 & & $0.006 \ddagger$ \\
\hline Tertile $2(\%)$ & & & 33 & & & .9 & & 33 & 8 & 32 & .5 & & 33 & & 33 & & \\
\hline Tertile $3(\%)$ & & & 34 & & & 3 & & 37 & 0 & 27 & 7 & & 37 & 6 & 27 & & \\
\hline Milk $(\mathrm{g} / \mathrm{d})^{*}$ & $245 \cdot 0$ & $418 \cdot 3$ & $245 \cdot 0$ & $418 \cdot 3$ & 244.0 & $418 \cdot 3$ & 0.308 & $287 \cdot 0$ & $402 \cdot 0$ & 244.0 & 471.5 & 0.001 & 305.0 & $402 \cdot 0$ & $244 \cdot 0$ & $470 \cdot 6$ & 0.001 \\
\hline Tertile $1(\%)$ & & & 48 & & & $\cdot 7$ & $0.858 \ddagger$ & 44 & & 57 & 3 & $0.002 \ddagger$ & 43 & & 57 & & $<0.001 \ddagger$ \\
\hline Tertile $2(\%)$ & & & 38 & & & $\cdot 1$ & & 42 & 1 & 30 & .3 & & 43 & & 29 & & \\
\hline Tertile $3(\%)$ & & & 13 & & & $\cdot 2$ & & 13 & 7 & 12 & 4 & & 13 & & 13 & & \\
\hline Yoghurt $(g / d)^{\star}$ & $53 \cdot 6$ & $107 \cdot 2$ & $53 \cdot 6$ & $107 \cdot 2$ & $53 \cdot 6$ & $107 \cdot 2$ & 0.377 & $53 \cdot 6$ & $107 \cdot 2$ & $53 \cdot 6$ & $107 \cdot 2$ & 0.663 & $53 \cdot 6$ & $107 \cdot 2$ & $53 \cdot 6$ & $107 \cdot 2$ & $0 \cdot 723$ \\
\hline Tertile $1(\%)$ & & & 34 & & & & $0 \cdot 249 \ddagger$ & 33 & & 32 & .5 & $0 \cdot 139 \ddagger$ & 32 & & 34 & & $0 \cdot 132 \ddagger$ \\
\hline Tertile $2(\%)$ & & & 25 & & & .9 & & 26 & 6 & 21 & .2 & & 27 & & 20 & & \\
\hline Tertile $3(\%)$ & & & 40 & & & $\cdot 7$ & & 39 & .6 & 46 & .4 & & 40 & 3 & 44 & & \\
\hline Cheese $(\mathrm{g} / \mathrm{d})^{*}$ & $12 \cdot 9$ & $21 \cdot 6$ & $12 \cdot 9$ & $20 \cdot 1$ & $12 \cdot 9$ & $21 \cdot 6$ & 0.609 & $12 \cdot 9$ & $19 \cdot 3$ & $12 \cdot 9$ & $21 \cdot 6$ & 0.425 & $12 \cdot 9$ & $19 \cdot 3$ & $12 \cdot 9$ & $21 \cdot 6$ & $0 \cdot 246$ \\
\hline Tertile 1 (\%) & & & 25 & & & $\cdot 8$ & $0 \cdot 712 \ddagger$ & 23 & 1 & 30 & $\cdot 3$ & $0.043 \ddagger$ & 23 & & 29 & & $0.076 \ddagger$ \\
\hline Tertile $2(\%)$ & & & 41 & & & $\cdot 2$ & & 44 & 2 & 35 & 8 & & 44 & & 36 & & \\
\hline Tertile $3(\%)$ & & & 33 & & & .0 & & 32 & 6 & 33 & 3.9 & & 32 & 9 & 33 & & \\
\hline $\operatorname{RTEC}(\mathrm{g} / \mathrm{d})^{\star}$ & $17 \cdot 1$ & $34 \cdot 3$ & $31 \cdot 4$ & $34 \cdot 3$ & $17 \cdot 1$ & $25 \cdot 7$ & $<0.001$ & $31 \cdot 4$ & $39 \cdot 3$ & $17 \cdot 1$ & $34 \cdot 3$ & 0.009 & $31 \cdot 4$ & $34 \cdot 3$ & $17 \cdot 1$ & $34 \cdot 3$ & 0.001 \\
\hline Tertile $1(\%)$ & & & 27 & & & $\cdot 2$ & $0.002 \ddagger$ & 27 & 3 & 33 & 3.6 & $0.093 \ddagger$ & 26 & & 34 & & $0.010 \ddagger$ \\
\hline Tertile $2(\%)$ & & & 35 & & & 5 & & 36 & 8 & 37 & .6 & & 36 & & 38 & & \\
\hline Tertile $3(\%)$ & & & 37 & & & $\cdot 2$ & & 35 & 9 & 28 & 3.8 & & 37 & 3 & 27 & 4 & \\
\hline Fruits $(g / d)^{*}$ & $215 \cdot 3$ & 308.9 & $223 \cdot 6$ & 298.9 & $207 \cdot 5$ & $323 \cdot 4$ & $0 \cdot 191$ & $212 \cdot 1$ & $270 \cdot 7$ & $220 \cdot 3$ & $341 \cdot 4$ & 0.668 & $214 \cdot 5$ & $292 \cdot 8$ & $216 \cdot 3$ & $332 \cdot 3$ & 0.946 \\
\hline Tertile $1(\%)$ & & & 31 & & & 9 & $0.086 \ddagger$ & 32 & 4 & 34 & 1.7 & $0.072 \ddagger$ & 32 & & 34 & & $0.437 \ddagger$ \\
\hline Tertile $2(\%)$ & & & 35 & & & 2 & & 36 & 6 & 28 & 3.5 & & 35 & & 30 & & \\
\hline Tertile $3(\%)$ & & & 33 & & & .9 & & 31 & 0 & 36 & 9 & & 32 & 2 & 34 & .8 & \\
\hline Vegetables $(\mathrm{g} / \mathrm{d})^{*}$ & $91 \cdot 5$ & $133 \cdot 7$ & $92 \cdot 2$ & $132 \cdot 2$ & $85 \cdot 8$ & $136 \cdot 1$ & 0.739 & $95 \cdot 1$ & $134 \cdot 6$ & $85 \cdot 5$ & $132 \cdot 7$ & $0 \cdot 153$ & $92 \cdot 7$ & $135 \cdot 1$ & $87 \cdot 5$ & $134 \cdot 2$ & $0 \cdot 231$ \\
\hline Tertile $1(\%)$ & & & 33 & & & 5 & $0.896 \ddagger$ & 32 & & 35 & 50 & $0.638 \ddagger$ & 31 & & 35 & & $0.676 \ddagger$ \\
\hline Tertile $2(\%)$ & & & 33 & & & .4 & & 33 & 3 & 33 & 3.6 & & 34 & & 32 & & \\
\hline Tertile 3 (\%) & & & 33 & & & 2 & & 34 & .5 & 31 & .4 & & 33 & .9 & 32 & & \\
\hline Vegetable soup $(\mathrm{g} / \mathrm{d})^{*}$ & $126 \cdot 4$ & $189 \cdot 7$ & $126 \cdot 4$ & $189 \cdot 7$ & $126 \cdot 4$ & $189 \cdot 7$ & 0.586 & $126 \cdot 4$ & $189 \cdot 7$ & $126 \cdot 4$ & $189 \cdot 7$ & 0.292 & $126 \cdot 4$ & $189 \cdot 7$ & $126 \cdot 4$ & $212 \cdot 1$ & 0.048 \\
\hline Tertile $1(\%)$ & & & 21 & & & $\cdot 1$ & $0.942 \ddagger$ & 19 & .7 & 23 & 3.7 & $0.440 \ddagger$ & 18 & & 25 & & $0.099 \ddagger$ \\
\hline Tertile $2(\%)$ & & & 43 & & & 3 & & 44 & 0 & 41 & 6 & & 44 & & 41 & .5 & \\
\hline Tertile 3 (\%) & & & 35 & & & 6 & & 36 & 3 & 34 & 1.7 & & 37 & & 33 & & \\
\hline Sweets and pastries $(\mathrm{g} / \mathrm{d})^{*}$ & $39 \cdot 7$ & $51 \cdot 2$ & $44 \cdot 2$ & $56 \cdot 1$ & $27 \cdot 5$ & $41 \cdot 0$ & $<0.001$ & $44 \cdot 1$ & $53 \cdot 2$ & $31 \cdot 4$ & $44 \cdot 7$ & $<0.001$ & $46 \cdot 1$ & $54 \cdot 4$ & $29 \cdot 5$ & $42 \cdot 8$ & $<0.001$ \\
\hline Tertile $1(\%)$ & & & 26 & & & $\cdot 1$ & $<0.001 \ddagger$ & 27 & 3 & 42 & .7 & $<0.001 \ddagger$ & 24 & .8 & 44 & & $<0.001 \ddagger$ \\
\hline Tertile $2(\%)$ & & & 35 & & & 1 & & 35 & 9 & 29 & 6 & & 36 & & 29 & & \\
\hline Tertile 3 (\%) & & & 37 & & & 8 & & 36 & 8 & 27 & .7 & & 38 & 8 & 25 & 8 & \\
\hline
\end{tabular}


It has been suggested that SES in childhood may influence health behaviours and, consequently, the predisposition to obesity $^{(55)}$. In the present study SES was assessed by measuring parental education and employment. After adjustment, only mother's education level in boys and father's employment status in girls remained significant predictors of abdominal obesity. A recent study with data from different countries found that higher maternal education was associated with more favourable growth in young children; that is, lower obesity and overweight in the UK and Sweden, and lower stunting and underweight in rural China ${ }^{(56)}$. Likewise, Koupil and Toivanen ${ }^{(57)}$ reported in a sample of 18-year-old Swedish men that prevalence of overweight and obesity decreased with higher maternal education. On the other hand, as recently proposed in a review, father's employment is also a probable early marker of the development of obesity in adulthood $^{(13)}$. In particular, the employment of fathers in 'low status', 'blue collar', 'unskilled' and 'manual' jobs is classically associated with increased risk of being obese as an adult ${ }^{(13)}$.

In our sample, boys with abdominal obesity were less active than their lean counterparts and physical activity seemed to be a negative predictor of abdominal obesity. Indeed, evidence suggests that low physical activity levels may play a role in the development of abdominal obesity in youth $^{(58)}$. A cross-sectional study with adolescents showed that WC was inversely associated with structured physical activity (outside school, $>140 \mathrm{~min} /$ week), independently of time spent on sedentary activities ${ }^{(59)}$. Likewise, Ortega et $a l^{(60)}$ found that children and adolescents with low levels of vigorous physical activity had higher odds of having high WC, when compared with those with high levels of vigorous physical activity.

Overweight and obese adolescents, as well as adolescents with abdominal obesity, presented lower intake of sweets and pastries. In addition, vegetable intake was positively associated with abdominal obesity in boys, whereas sweets and pastries intake was negatively associated with the same in girls. There is no evidence that a high intake of vegetables is associated with higher risk of obesity in children and adolescents. It is described that the wide variability in methods of cooking and preparing vegetables may contribute to differences in energy density and macronutrient composition, which may modify the effects of vegetables on body weight ${ }^{(61,62)}$. On one hand, these findings could be related to the effect described earlier, that obese adolescents under-report their food intake more than their lean counterparts ${ }^{(63,64)}$. Furthermore, foods that are more socially desirable and approved may be overestimated, and the opposite may also occur ${ }^{(65)}$. On the other hand, these results may also be due to the crosssectional design of the present study, which might have distorted the temporal relationship between diet and weight. Adolescents who are overweight and obese probably decrease their intake of highly energy-dense foods, in 
Table 5 Univariate associations between overweight/obesity and abdominal obesity (WC $\geq \mathrm{P} 90$ or WHtR $\geq 0.500$ ) according to food group intakes, physical activity and socio-economic status: adolescent boys and girls aged 15-18 years, Azorean Archipelago, Portugal, 2008

\begin{tabular}{|c|c|c|c|c|c|c|c|c|c|c|c|c|c|c|c|c|}
\hline & \multicolumn{8}{|c|}{ Boys } & \multicolumn{8}{|c|}{ Girls } \\
\hline & BMI & \multirow[b]{2}{*}{$P$} & \multicolumn{2}{|r|}{ WC } & \multirow[b]{2}{*}{$P$} & \multicolumn{2}{|r|}{ WHtR } & \multirow[b]{2}{*}{$P$} & BMI & \multirow[b]{2}{*}{$P$} & \multicolumn{2}{|r|}{ WC } & \multirow[b]{2}{*}{$P$} & \multicolumn{2}{|r|}{ WHtR } & \multirow[b]{2}{*}{$P$} \\
\hline & $95 \% \mathrm{Cl}$ & & OR & $95 \% \mathrm{Cl}$ & & OR & $95 \% \mathrm{Cl}$ & & $95 \% \mathrm{Cl}$ & & OR & $95 \% \mathrm{Cl}$ & & OR & $95 \% \mathrm{Cl}$ & \\
\hline \multicolumn{17}{|l|}{ Father's employment } \\
\hline High & Reference & $0.374^{*}$ & & Reference & $0.372^{*}$ & & Reference & $0.358^{*}$ & Reference & $0.086^{*}$ & & Reference & $0.002^{*}$ & & Reference & $0.027^{\star}$ \\
\hline Medium & $1.6720 .723,3 \cdot 712$ & & $2 \cdot 048$ & $30 \cdot 688,6 \cdot 100$ & & $2 \cdot 082$ & $0 \cdot 772,5 \cdot 615$ & & $1.040 \quad 0.571,1.891$ & & $1 \cdot 429$ & $0.775,2 \cdot 633$ & & $1 \cdot 281$ & $0.718,2 \cdot 285$ & \\
\hline Low & $1.3630 .611,3.043$ & & 1.964 & $40 \cdot 658,5 \cdot 864$ & & $2 \cdot 257$ & $0.838,6.078$ & & $1 \cdot 1440.635,2 \cdot 059$ & & $2 \cdot 130$ & $1 \cdot 170,3 \cdot 880$ & & 1.766 & $1 \cdot 000,3 \cdot 117$ & \\
\hline No relationship with employment & $0.8610 .252,2 \cdot 944$ & & 0.783 & $30.132,4 \cdot 623$ & & 1.333 & $0.322,5.526$ & & $2 \cdot 3201 \cdot 061,5 \cdot 076$ & & 3.496 & $1 \cdot 574,7 \cdot 764$ & & 2.594 & $1 \cdot 195,5 \cdot 632$ & \\
\hline \multicolumn{17}{|c|}{ Mother's employment } \\
\hline High & Reference & $0.650^{*}$ & & Reference & $0 \cdot 208^{*}$ & & Reference & $0.087^{*}$ & Reference & $0 \cdot 140^{*}$ & & Reference & $0.001^{*}$ & & Reference & $0.004^{*}$ \\
\hline Medium & $1.2620 .659,2 \cdot 416$ & & 1.464 & $4 \quad 0.640,3 \cdot 347$ & & $2 \cdot 313$ & $1 \cdot 029,5 \cdot 201$ & & $0.6380 .353,1.153$ & & 0.733 & $0 \cdot 407,1 \cdot 320$ & & 0.649 & $0 \cdot 366,1 \cdot 152$ & \\
\hline Low & $0.9630 .501,1.853$ & & $1 \cdot 133$ & $3 \quad 0 \cdot 490,2 \cdot 617$ & & 1.626 & $0.716,3.690$ & & $0.8330 .468,1.485$ & & $1 \cdot 378$ & $0 \cdot 776,2 \cdot 444$ & & $1 \cdot 129$ & $0.644,1.980$ & \\
\hline No relationship with employment & $1.0980 .252,4 \cdot 780$ & & 4.083 & $0.939,17.749$ & & 4.083 & $0.939,17.749$ & & $2.0450 .496,8.443$ & & 3.273 & $0.742,14.434$ & & 2.462 & $0.561,10.807$ & \\
\hline Father's education & & & & & & & & & & & & & & & & \\
\hline College/university & Reference & $0.987^{\star}$ & & Reference & $0.341^{*}$ & & Reference & $0 \cdot 120^{*}$ & Reference & $0 \cdot 414^{*}$ & & Reference & $0.019^{*}$ & & Reference & $0 \cdot 103^{*}$ \\
\hline Secondary & $1.0160 .397,2 \cdot 603$ & & $2 \cdot 625$ & $0.559,12 \cdot 321$ & & 3.574 & $0 \cdot 776,16 \cdot 460$ & & $1.582 \quad 0.643,3.892$ & & $2 \cdot 065$ & $0 \cdot 813,5 \cdot 241$ & & 1.793 & $0.755,4.257$ & \\
\hline Mandatory or less & $1 \cdot 0530 \cdot 446,2 \cdot 484$ & & $2 \cdot 971$ & $0.682,12.940$ & & $4 \cdot 430$ & $1 \cdot 024,19 \cdot 155$ & & $1.7250 .763,3.896$ & & $2 \cdot 966$ & $1 \cdot 266,6 \cdot 947$ & & $2 \cdot 229$ & $1 \cdot 019,4.875$ & \\
\hline Mother's education & & & & & & & & & & & & & & & & \\
\hline College/university & Reference & $0.978^{*}$ & & Reference & $0.403^{*}$ & & Reference & $0 \cdot 118^{*}$ & Reference & $0.912^{*}$ & & Reference & $0 \cdot 125^{*}$ & & Reference & $0 \cdot 250^{*}$ \\
\hline Secondary & $1 \cdot 0140 \cdot 485,2 \cdot 122$ & & 1.412 & $20.517,3.858$ & & $2 \cdot 338$ & $0 \cdot 889,6 \cdot 150$ & & $0.950 \quad 0.489,1.845$ & & 1.026 & $0.525,2.005$ & & 0.987 & $0.512,1.900$ & \\
\hline Mandatory or less & $0.963 \quad 0.495,1.874$ & & 1.786 & $0.718,4 \cdot 444$ & & 2.586 & $1.050,6 \cdot 367$ & & $0.8950 .501,1.598$ & & 1.498 & $0 \cdot 836,2 \cdot 683$ & & 1.352 & $0.764,2 \cdot 393$ & \\
\hline PAI & $0.6700 .402,1 \cdot 117$ & $0.125^{*}$ & 0.601 & $0.333,1.083$ & $0.090^{*}$ & 0.583 & $0.341,0.997$ & $0.049^{*}$ & $1.0140 .739,1.391$ & $0.931^{*}$ & 0.843 & $0 \cdot 622,1 \cdot 141$ & $0.269^{*}$ & 0.982 & $0 \cdot 729,1 \cdot 324$ & $0.907^{*}$ \\
\hline Total dairy & & & & & & & & & & & & & & & & \\
\hline Tertile 1 & Reference & $0.481+$ & & Reference & $0.698+$ & & Reference & $0.673+$ & Reference & $0.412 \dagger$ & & Reference & $0.002 \dagger$ & & Reference & $0.001+$ \\
\hline Tertile 2 & $0.8290 .515,1.333$ & & 0.931 & $10.524,1.655$ & & 0.824 & $0.490,1.384$ & & $0.9620 .655,1.413$ & & 0.705 & $0.488,1.018$ & & 0.734 & $0.510,1.056$ & \\
\hline Tertile 3 & $0.8450 .531,1.346$ & & 0.893 & $30.506,1.578$ & & 0.896 & $0.542,1.483$ & & $0.850 \quad 0.576,1.253$ & & 0.549 & $0.378,0.799$ & & 0.547 & $0.378,0.792$ & \\
\hline Milk & & & & & & & & & & & & & & & & \\
\hline Tertile 1 & Reference & $0.985+$ & & Reference & $0.805 t$ & & Reference & $0.866+$ & Reference & $0.730+$ & & Reference & $0.009+$ & & Reference & $0.010+$ \\
\hline Tertile 2 & $0.8790 .562,1.376$ & & 0.887 & $70.515,1.528$ & & 0.862 & $0.529,1.404$ & & $0.9080 .644,1.279$ & & 0.555 & $0.397,0.775$ & & 0.500 & $0.359,0.697$ & \\
\hline Tertile 3 & $1 \cdot 0430 \cdot 636,1 \cdot 113$ & & 0.951 & $0.518,1.747$ & & 0.991 & $0.578,1.700$ & & $0.9650 .592,1.573$ & & $0 \cdot 701$ & $0.437,1.124$ & & 0.772 & $0.487,1.225$ & \\
\hline Yoghurt & & & & & & & & & & & & & & & & \\
\hline Tertile 1 & Reference & $0.534 \dagger$ & & Reference & $0.448+$ & & Reference & $0.818 \dagger$ & Reference & $0 \cdot 119+$ & & Reference & $0.226+$ & & Reference & $0.704+$ \\
\hline Tertile 2 & $0.6270 .365,1.078$ & & & $10.364,1.350$ & & 1.031 & $0.588,1.805$ & & $1.034 \quad 0.673,1.589$ & & 0.824 & $0.548,1.248$ & & 0.716 & $0.478,1.072$ & \\
\hline Tertile 3 & $0.8930 .581,1.372$ & & 0.845 & $5 \quad 0.500,1.428$ & & 1.057 & $0.659,1.695$ & & $1.3280 .920,1.918$ & & $1 \cdot 218$ & $0.859,1.728$ & & 1.047 & $0.742,1.477$ & \\
\hline Cheese & & & & & & & & & & & & & & & & \\
\hline Tertile 1 & Reference & $0.193 t$ & & Reference & $0.637+$ & & Reference & $0.534 t$ & Reference & $0.643+$ & & Reference & $0.323+$ & & Reference & $0.292 \dagger$ \\
\hline Tertile 2 & $0.7010 .429,1 \cdot 145$ & & 0.858 & $3 \quad 0.472,1.560$ & & 0.921 & $0.541,1.570$ & & $0.848 \quad 0.572,1.257$ & & 0.618 & $0.423,0.903$ & & 0.649 & $0.446,0.944$ & \\
\hline Tertile 3 & $0 \cdot 7180.449,1 \cdot 151$ & & 0.864 & $40.486,1.535$ & & 0.848 & $30.505,1.426$ & & $0.8980 .596,1.354$ & & 0.795 & $0.537,1.175$ & & 0.788 & $0.534,1.163$ & \\
\hline RTEC & & & & & & & & & & & & & & & & \\
\hline Tertile 1 & Reference & $0.001+$ & & Reference & $0.005 t$ & & Reference & $<0.001+$ & Reference & $0.001 t$ & & Reference & $0.030+$ & & Reference & $0.003+$ \\
\hline Tertile 2 & $0.7690 .508,1.164$ & & 0.764 & $4 \quad 0.467,1.250$ & & 0.605 & $0.389,0.941$ & & $0.8790 .603,1.282$ & & 0.831 & $0.575,1.201$ & & 0.800 & $0.556,1.152$ & \\
\hline Tertile 3 & $0.2080 .084,0.514$ & & $0 \cdot 131$ & $10.030,0.564$ & & $0 \cdot 164$ & $0.056,0.477$ & & $0.4990 .331,0.753$ & & 0.654 & $0.445,0.960$ & & 0.560 & $0.383,0.820$ & \\
\hline Fruits & & & & & & & & & & & & & & & & \\
\hline Tertile 1 & Reference & $0.127 \dagger$ & & Reference & $0.393+$ & & Reference & $0.250 t$ & Reference & $0.374 t$ & & Reference & $0.570+$ & & Reference & $0.926+$ \\
\hline Tertile 2 & $1.2250 \cdot 716,1 \cdot 986$ & & $1 \cdot 136$ & 6. $0.631,2.045$ & & 1.067 & $0.630,1.807$ & & $0.6450 .436,0.953$ & & 0.728 & $0.499,1.060$ & & 0.819 & $0.567,1.182$ & \\
\hline Tertile 3 & $1.4490 .900,2 \cdot 330$ & & 1.285 & $0.722,2.288$ & & 1.346 & $0.807,2.245$ & & $0.8440 .577,1.235$ & & $1 \cdot 111$ & $0.770,1.603$ & & 1.017 & $0.707,1.464$ & \\
\hline Vegetables & & & & & & & & & & & & & & & & \\
\hline Tertile 1 & Reference & $0.018+$ & & Reference & $0.031+$ & & Reference & $0.040+$ & Reference & $0.767+$ & & Reference & $0.344 t$ & & & $0.455+$ \\
\hline Tertile 2 & $1.8861 .153,3.083$ & & $2 \cdot 322$ & $21 \cdot 243,4 \cdot 337$ & & $2 \cdot 453$ & $31 \cdot 422,4 \cdot 233$ & & $1.0330 .703,1.518$ & & 0.925 & $0.640,1.338$ & & 0.864 & $0.600,1 \cdot 245$ & \\
\hline Tertile 3 & $1.8361 .122,3.004$ & & $2 \cdot 076$ & $1 \cdot 103,3.907$ & & 1.862 & $1 \cdot 064,3.258$ & & $0.9430 .639,1.391$ & & 0.836 & $0.576,1.212$ & & 0.870 & $0.604,1.254$ & \\
\hline
\end{tabular}




\begin{tabular}{|c|c|c|c|c|c|c|c|c|c|c|c|c|c|c|c|c|c|c|}
\hline & \multicolumn{9}{|c|}{ Boys } & \multicolumn{9}{|c|}{ Girls } \\
\hline & \multicolumn{2}{|r|}{$\mathrm{BMI}$} & \multirow[b]{2}{*}{$P$} & \multicolumn{2}{|r|}{ WC } & \multirow[b]{2}{*}{$P$} & \multicolumn{2}{|r|}{ WHtR } & \multirow[b]{2}{*}{$P$} & \multicolumn{2}{|r|}{ BMI } & \multirow[b]{2}{*}{$P$} & \multicolumn{2}{|r|}{ WC } & \multirow[b]{2}{*}{$P$} & \multicolumn{2}{|r|}{ WHtR } & \multirow[b]{2}{*}{$P$} \\
\hline & OR & $95 \% \mathrm{Cl}$ & & OR & $95 \% \mathrm{Cl}$ & & OR & $95 \% \mathrm{Cl}$ & & OR & $95 \% \mathrm{Cl}$ & & OR & $95 \% \mathrm{Cl}$ & & OR & $95 \% \mathrm{Cl}$ & \\
\hline \multicolumn{19}{|c|}{ Vegetable soup } \\
\hline Tertile 1 & & ference & $0.951 \dagger$ & & Reference & $0.480+$ & & Reference & $0.462+$ & $\mathrm{R}$ & eference & $0.811+$ & & Reference & $0.318+$ & & Reference & $0.062 \dagger$ \\
\hline Tertile 2 & 0.962 & $0.561,1.651$ & & 0.920 & $0.484,1.751$ & & 1.050 & $0.594,1.856$ & & 0.981 & $0.645,1 \cdot 492$ & & 0.785 & $0.527,1 \cdot 168$ & & 0.689 & $0.465,1 \cdot 021$ & \\
\hline Tertile 3 & 1.011 & $0 \cdot 652,1 \cdot 568$ & & 0.827 & $0.486,1.407$ & & 0.843 & $0.522,1.361$ & & $1 \cdot 044$ & $0.677,1 \cdot 608$ & & 0.791 & $0.524,1 \cdot 194$ & & 0.658 & $0.438,0.989$ & \\
\hline \multicolumn{19}{|c|}{ Sweets and pastries } \\
\hline Tertile 1 & & ference & $0.028+$ & & Reference & $0.013+$ & & Reference & $0.004 t$ & $\mathrm{R}$ & eference & $<0.001+$ & & Reference & $<0.001+$ & & Reference & $<0.001 \dagger$ \\
\hline Tertile 2 & $0 \cdot 818$ & $0.516,1.295$ & & 0.759 & $0.441,1.308$ & & 0.824 & $0.506,1.342$ & & 0.464 & $0.317,0.681$ & & 0.527 & $0.364,0.764$ & & 0.448 & $0.310,0.648$ & \\
\hline \multirow{2}{*}{\multicolumn{19}{|c|}{ Fast food }} \\
\hline & & & & & & & & & & & & & & & & & & \\
\hline Tertile 1 & & ference & $0.420 t$ & & Reference & $0.133+$ & & Reference & $0.374 t$ & & eference & $0.012+$ & & Reference & $0.370 t$ & & Reference & $0.344 t$ \\
\hline Tertile 2 & 1.335 & $0 \cdot 831,2 \cdot 145$ & & 1.033 & $0.592,1.803$ & & $1 \cdot 254$ & $0.752,2 \cdot 091$ & & 0.806 & $0.551,1 \cdot 180$ & & 0.989 & $0.682,1.433$ & & 0.991 & $10.687,1 \cdot 430$ & \\
\hline Tertile 3 & 0.830 & $0.498,1.382$ & & 0.622 & $0.333,1 \cdot 162$ & & 0.790 & $0.452,1.379$ & & 0.605 & $0.407,0.899$ & & 0.843 & $30.579,1.229$ & & 0.838 & $30.578,1.214$ & \\
\hline \multicolumn{19}{|c|}{ 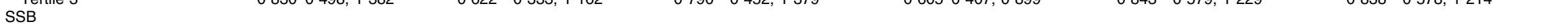 } \\
\hline Tertile 1 & & ference & $0.678+$ & & Reference & $0.788+$ & & Reference & $0.683+$ & & eference & $0.003+$ & & Reference & $0.039+$ & & Reference & $0.030 t$ \\
\hline Tertile 2 & 0.704 & $0.437,1 \cdot 136$ & & 0.787 & $0.440,1.406$ & & 0.822 & $0.491,1.377$ & & 0.593 & $0 \cdot 402,0 \cdot 875$ & & 0.571 & $0.391,0.833$ & & 0.673 & $30.465,0.975$ & \\
\hline Tertile 3 & 0.906 & $0.570,1.439$ & & 0.926 & $0.527,1.624$ & & 0.900 & $0.542,1.494$ & & 0.565 & $0.385,0.828$ & & 0.680 & $0.472,0.979$ & & 0.668 & $3 \quad 0.465,0.960$ & \\
\hline
\end{tabular}

WC, waist circumference; P90, 90th percentile; WHtR, waist-to-height ratio; PAl, physical activity index; RTEC, ready-to-eat cereals; SSB, sugar-sweetened beverages

Each variable enters in the model separately; the reference group in all food group items is tertile 1 while for PAl the reference is low activity.

$P$ value for heterogeneity.

Boys. Dairy: tertile 1, $\leq 297.57 \mathrm{~g} / \mathrm{d}$; tertile 2, 297.58-676.43 g/d; tertile 3, $\geq 676 \cdot 44 \mathrm{~g} / \mathrm{d}$. Milk: tertile 1, $\leq 244.00 \mathrm{~g} / \mathrm{d}$; tertile 2, 244.01-610.00 g/d; tertile 3, $\geq 610 \cdot 01 \mathrm{~g} / \mathrm{d}$. Yoghurt: tertile 1, $\leq 17 \cdot 85 \mathrm{~g} / \mathrm{d}$; tertile 2, 17.86-98.21 g/d; tertile

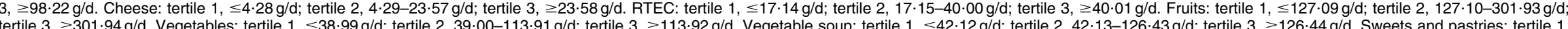
$\leq 28.26 \mathrm{~g} / \mathrm{d}$; tertile $2,28.27-66.54 \mathrm{~g} / \mathrm{d}$; tertile $3, \geq 66.55 \mathrm{~g} / \mathrm{d}$. Fast food: tertile $1, \leq 31.62 \mathrm{~g} / \mathrm{d}$; tertile $2,31.63-66.47 \mathrm{~g} / \mathrm{d}$; tertile $3 \geq 66.48 \mathrm{~g} / \mathrm{d}$. SSB: tertile $1 \leq 158.84 \mathrm{~m} / \mathrm{d}$; tertile $2,158.85-418.26 \mathrm{ml} / \mathrm{d}$; tertile $3, \geq 418.29 \mathrm{~m} / \mathrm{d}$.

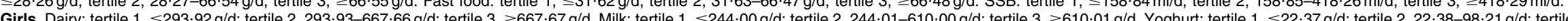
$3, \geq 98.22 \mathrm{~g} / \mathrm{d}$. Cheese: tertile $1, \leq 4 \cdot 28 \mathrm{~g} / \mathrm{d}$; tertile $2,4 \cdot 29-13.00 \mathrm{~g} / \mathrm{d}$; tertile $3, \geq 13.01 \mathrm{~g} / \mathrm{d}$. RTEC: tertile $1, \leq 17 \cdot 14 \mathrm{~g} / \mathrm{d}$; tertile $2,17 \cdot 15-31 \cdot 43 \mathrm{~g} / \mathrm{d}$; tertile $3, \geq 31 \cdot 44 \mathrm{~g} / \mathrm{d}$. Fruits: tertile $1, \leq 141 \cdot 05 \mathrm{~g} / \mathrm{d}$; tertile $2,141 \cdot 06-321 \cdot 30 \mathrm{~g} / \mathrm{d}$; tertile $3, \geq 321.31 \mathrm{~g} / \mathrm{d}$. Vegetables: tertile $1, \leq 54.25 \mathrm{~g} / \mathrm{d}$; tertile $2,54 \cdot 26-130.01 \mathrm{~g} / \mathrm{d}$; tertile $3, \geq 130.02 \mathrm{~g} / \mathrm{d}$. Vegetable soup: tertile $1, \leq 42.12 \mathrm{~g} / \mathrm{d}$; tertile $2,42 \cdot 13-231 \cdot 78 \mathrm{~g} / \mathrm{d}$; tertile $3, \geq 231.79 \mathrm{~g} / \mathrm{d}$. Sweets and pastries: tertile 1, $\leq 24 \cdot 76 \mathrm{~g} / \mathrm{d}$; tertile $2,24 \cdot 77-56.62 \mathrm{~g} / \mathrm{d}$; tertile $3, \geq 56 \cdot 63 \mathrm{~g} / \mathrm{d}$. Fast food: tertile $1, \leq 31 \cdot 01 \mathrm{~g} / \mathrm{d}$; tertile $2,31 \cdot 02-49 \cdot 45 \mathrm{~g} / \mathrm{d}$; tertile $3, \geq 49 \cdot 46 \mathrm{~g} / \mathrm{d}$. SSB: tertile $1, \leq 94 \cdot 24 \mathrm{ml} / \mathrm{d}$; tertile $2,94 \cdot 25-300 \cdot 42 \mathrm{ml} / \mathrm{d}$; tertile $3, \geq 300 \cdot 43 \mathrm{ml} / \mathrm{d}$. 
Table 6 Adjusted odds ratios for overweight/obesity and abdominal obesity (WC $\geq \mathrm{P} 90$ or WHtR $\geq 0.500$ ) according to food group intakes, physical activity and socio-economic status that remained after the conditional stepwise method: adolescent boys and girls aged 15-18 years, Azorean Archipelago, Portugal, 2008

\begin{tabular}{|c|c|c|c|c|c|c|c|c|c|c|c|c|c|c|c|c|c|c|}
\hline & \multicolumn{9}{|c|}{ Boys } & \multicolumn{9}{|c|}{ Girls } \\
\hline & \multicolumn{2}{|r|}{ BMI } & \multirow[b]{2}{*}{$P$} & \multicolumn{2}{|r|}{ WC } & \multirow[b]{2}{*}{$P$} & \multicolumn{2}{|r|}{ WHtR } & \multirow[b]{2}{*}{$P$} & \multicolumn{2}{|r|}{ BMI } & \multirow[b]{2}{*}{$P$} & \multicolumn{2}{|r|}{ WC } & \multirow[b]{2}{*}{$P$} & \multicolumn{2}{|r|}{ WHtR } & \multirow[b]{2}{*}{$P$} \\
\hline & OR & $95 \% \mathrm{Cl}$ & & OR & $95 \% \mathrm{Cl}$ & & OR & $95 \% \mathrm{Cl}$ & & OR & $95 \% \mathrm{Cl}$ & & OR & $95 \% \mathrm{Cl}$ & & OR & $95 \% \mathrm{Cl}$ & \\
\hline \multicolumn{19}{|l|}{ Father's employment } \\
\hline High & - & & & - & & & - & & & - & & & $R$ & eference & $0.066^{*}$ & & eference & $0 \cdot 181^{\star}$ \\
\hline $\begin{array}{l}\text { Meaw } \\
\text { Low }\end{array}$ & & & & & & & & & & & & & $\begin{array}{l}1.316 \\
1.883\end{array}$ & $\begin{array}{l}0.642,2.700 \\
0.905,3.915\end{array}$ & & $\begin{array}{l}1.295 \\
1.732\end{array}$ & $\begin{array}{l}0.646,2.598 \\
0.849,3.533\end{array}$ & \\
\hline No relationship with employment & & & & & & & & & & & & & 2.672 & $1.062,6.726$ & & $2 \cdot 261$ & $0.906,5 \cdot 640$ & \\
\hline \multicolumn{19}{|l|}{ Mother's employment } \\
\hline High & - & & & - & & & - & & & & Reference & $0 \cdot 075^{\star}$ & & eference & $0 \cdot 017^{\star}$ & & eference & $0 \cdot 012^{*}$ \\
\hline Medium & & & & & & & & & & 0.759 & $0.396,1.454$ & & 0.621 & $0 \cdot 316,1 \cdot 221$ & & 0.523 & $0.268,1.019$ & \\
\hline Low & & & & & & & & & & $1 \cdot 170$ & $0 \cdot 616,2 \cdot 221$ & & $1 \cdot 115$ & $0.558,2.228$ & & 0.939 & $0.472,1.867$ & \\
\hline \multirow{2}{*}{\multicolumn{19}{|c|}{ Mother's education }} \\
\hline & & & & & & & & & & & & & & & & & & \\
\hline College/university & - & & & - & & & & Reference & $0.063^{\star}$ & - & & & - & & & - & & \\
\hline $\begin{array}{l}\text { Secondary } \\
\text { Mandatory or less }\end{array}$ & & & & & & & $\begin{array}{l}3.054 \\
3.172\end{array}$ & $\begin{array}{l}1 \cdot 085,8.596 \\
1.200,8 \cdot 382\end{array}$ & & & & & & & & & & \\
\hline $\begin{array}{l}\text { Mandatory or less } \\
\text { PAI }\end{array}$ & & & & & & & 0.454 & $\begin{array}{l}1.234,0.880 \\
0.0 .830\end{array}$ & $0.019^{\star}$ & & & & & & & & & \\
\hline \multicolumn{19}{|c|}{$0.454 \quad 0.234,0.8800 .019^{n}$} \\
\hline Tertile 1 & - & & & - & & & - & & & - & & & & eference & $0.198+$ & & ieference & $0 \cdot 151 t$ \\
\hline Tertile 2 & & & & & & & & & & & & & $0 \cdot 701$ & $0.458,1.072$ & & 0.770 & $0.504,1 \cdot 176$ & \\
\hline Tertile 3 & & & & & & & & & & & & & 0.749 & $0.478,1 \cdot 173$ & & 0.720 & $0 \cdot 460,1 \cdot 127$ & \\
\hline \multicolumn{19}{|c|}{$07<0,0400$, TILr } \\
\hline Tertile 1 & - & & & - & & & - & & & - & & & & eference & $0.373+$ & & leference & $0 \cdot 183 t$ \\
\hline Tertile 2 & & & & & & & & & & & & & 0.636 & $0.434,0.932$ & & 0.534 & $0.364,0.762$ & \\
\hline Tertile 3 & & & & & & & & & & & & & 1.025 & $0.591,1.778$ & & 0.989 & $0.573,1.707$ & \\
\hline \multicolumn{19}{|l|}{ RTEC } \\
\hline Tertile 1 & \multicolumn{2}{|c|}{ Reference } & \multirow[t]{3}{*}{$0.004 t$} & & eference & $0.046+$ & & Reference & $0.002 \dagger$ & & Reference & $0 \cdot 116 t$ & - & & & & & \\
\hline Tertile 2 & 0.743 & $0.468,1 \cdot 179$ & & 0.894 & $0.520,1.539$ & & 0.576 & $0 \cdot 325,1 \cdot 022$ & & 1.031 & $0.672,1.581$ & & & & & & & \\
\hline Tertile 3 & 0.174 & $0.060,0.502$ & & 0.119 & $0.022,0.647$ & & 0.104 & $0.024,0.456$ & & 0.654 & $0.398,1.076$ & & & & & & & \\
\hline Vegetables & & & & & & & & & & & & & & & & & & \\
\hline Tertile 1 & & eference & $0.112 \dagger$ & & eference & $0.007 t$ & & Reference & $0.025 t$ & - & & & - & & & - & & \\
\hline Tertile 2 & 1.599 & $0.942,2 \cdot 716$ & & 2.299 & $1 \cdot 174,4.500$ & & $2 \cdot 225$ & $1 \cdot 117,4 \cdot 434$ & & & & & & & & & & \\
\hline Tertile 3 & 1.531 & $0.818,2 \cdot 867$ & & $2 \cdot 849$ & $1.271,6.386$ & & $2 \cdot 326$ & $1 \cdot 016,5 \cdot 327$ & & & & & & & & & & \\
\hline Sweets and pastries & & & & & & & & & & & & & & & & & & \\
\hline Tertile 1 & - & & & - & & & - & & & & Reference & $0.002 t$ & & ieference & $0.003+$ & & Seference & $<0.001+$ \\
\hline $\begin{array}{l}\text { Tertile } 2 \\
\text { Tertile } 3\end{array}$ & & & & & & & & & & $\begin{array}{l}0.509 \\
0.465\end{array}$ & $\begin{array}{l}0.330,0.786 \\
0.279,0.772\end{array}$ & & $\begin{array}{l}0.533 \\
0.487\end{array}$ & $\begin{array}{r}0.346,0.820 \\
0.296,0.800\end{array}$ & & $\begin{array}{l}0.436 \\
0.354\end{array}$ & $\begin{array}{l}0.283,0.671 \\
0.215,0.583\end{array}$ & \\
\hline
\end{tabular}

WC, waist circumference; P90, 90th percentile; WHtR, waist-to-height ratio; PAI, physical activity index; RTEC, ready-to-eat cereals.

Odds ratios adjusted for age, maturation, total energy intake (kJ/kcal), low-energy reporters and dietary fibre $(\mathrm{g} / 4184 \mathrm{~kJ}(1000 \mathrm{kcal}))$. For total dairy products, milk, yoghurt and cheese do not enter in the model. Significant results are shown in bold font.

${ }^{\star} P$ value for heterogeneity.

$+P$ value for trend.

Boys. RTEC: tertile 1, $\leq 17 \cdot 14 \mathrm{~g} / \mathrm{d}$; tertile 2, $17 \cdot 15-40.00 \mathrm{~g} / \mathrm{d}$; tertile $3, \geq 40.01 \mathrm{~g} / \mathrm{d}$. Vegetables: tertile $1, \leq 38.99 \mathrm{~g} / \mathrm{d}$; tertile 2, 39.00-113.91 g/d; tertile 3, $\geq 113.92 \mathrm{~g} / \mathrm{d}$. Sweets and pastries: tertile 1, $\leq 28 \cdot 26 \mathrm{~g} / \mathrm{d}$; tertile 2 , $28 \cdot 27-66 \cdot 54 \mathrm{~g} / \mathrm{d}$; tertile $3, \geq 66 \cdot 55 \mathrm{~g} / \mathrm{d}$.

Girls. Dairy: tertile $1, \leq 293.92 \mathrm{~g} / \mathrm{d}$; tertile 2, 293.93-667.66 g/d; tertile $3, \geq 667.67 \mathrm{~g} / \mathrm{d}$. Milk: tertile $1, \leq 244.00 \mathrm{~g} / \mathrm{d}$; tertile $2,244 \cdot 01-610.00 \mathrm{~g} / \mathrm{d}$; tertile 3, $\geq 610 \cdot 01 \mathrm{~g} / \mathrm{d}$. RTEC: tertile 1, $\leq 17 \cdot 14 \mathrm{~g} / \mathrm{d}$; tertile $2,17 \cdot 15-31 \cdot 43 \mathrm{~g} / \mathrm{d}$; tertile $3, \geq 31 \cdot 44 \mathrm{~g} / \mathrm{d}$. Sweets and pastries: tertile $1, \leq 24 \cdot 76 \mathrm{~g} / \mathrm{d}$; tertile $2,24 \cdot 77-56 \cdot 62 \mathrm{~g} / \mathrm{d}$; tertile $3, \geq 56 \cdot 63 \mathrm{~g} / \mathrm{d}$. 
order to lose weight ${ }^{(64)}$. For instance, girls are more likely to avoid high sugar and fat intakes since they usually demonstrate a greater concern for their body image and weight control than boys ${ }^{(66)}$.

In the current study, various measures of obesity had differential associations with food group intakes, physical activity and SES. Therefore, it may be important to use different measures of obesity, because the association of each measurement with health risks seems to be distinct. BMI is correlated with body fat, which, in excessive amounts, is related to metabolic complications, but BMI is also associated with lean mass, which may lead to some misclassification $^{(67)}$. There is evidence that WC is associated with visceral adipose tissue and is an independent risk factor for insulin resistance, hyperinsulinaemia, dyslipidaemia and hypertension in youth ${ }^{(23,68,69)}$. On the other hand, WHtR has been shown to be superior in its ability to predict CVD risk factors, compared with either BMI or percentage body fat, in children ${ }^{(22,70,71)}$

Some limitations of the present study should be acknowledged. As in every cross-sectional study, conclusions related to cause and effect cannot be drawn. The abdominal obesity measures used in the study are indirect estimates of visceral fat, and there are some sophisticated methods of accurately measuring visceral fat, such as MRI and dual-energy X-ray absorptiometric densitometry. However, such techniques cannot feasibly be applied in large epidemiological studies due to their complexity, timeconsuming nature and expense. Finally, with self-reported physical activity and dietary intake data, one cannot rule out some reporting bias, although both questionnaires have been tested previously ${ }^{(27,31)}$.

\section{Conclusion}

We found that ready-to-eat cereals in boys and milk in girls were negative predictors of overweight/obesity or abdominal obesity. It was also seen physical activity seems to be negatively associated with abdominal obesity in boys. Moreover, mother's education level in boys and father's employment status in girls were positive predictors of abdominal obesity. In addition, we also reported that different measures of obesity have distinct associations with food group intakes and SES. Thus, prospective and randomized clinical investigations are needed to examine the roles of food group intakes, physical activity and SES in the development of obesity, as assessed by different measures.

\section{Acknowledgements}

Sources of funding: This study was supported by Fundação para a Ciência e Tecnologia (FCT) - Ministério da Ciência, Tecnologia e Ensino Superior (MCTES; grant numbers BPD/65180/2009, BD/44422/2008, PEst-OE/SAU/UI0617/ 2011 and SFRH/BPD/50002/2009) and by the Azorean
Government. All funders had no role in the design, analysis or writing of this article. Conflicts of interest: The authors declare no conflicts of interest. Authors' contributions: S.A., R.S. and P.M. made substantial contributions to conception and design of data. S.A. and R.S. carried out the data collection, statistical analysis, interpretation of the data, and wrote the manuscript. C.A., P.C.S. and J.M. were involved in drafting the manuscript. All authors read and approved the final manuscript.

\section{References}

1. Lobstein T, Baur L \& Uauy R (2004) Obesity in children and young people: a crisis in public health. Obes Rev $\mathbf{5}$, Suppl. 1, 4-104.

2. Guo SS, Wu W, Chumlea WC et al. (2002) Predicting overweight and obesity in adulthood from body mass index values in childhood and adolescence. Am J Clin Nutr 76, 653-658.

3. de Koning L, Merchant AT, Pogue J et al. (2007) Waist circumference and waist-to-hip ratio as predictors of cardiovascular events: meta-regression analysis of prospective studies. Eur Heart J 28, 850-856.

4. Ashwell M \& Hsieh SD (2005) Six reasons why the waist-toheight ratio is a rapid and effective global indicator for health risks of obesity and how its use could simplify the international public health message on obesity. Int J Food Sci Nutr 56, 303-307.

5. Page A, Cooper AR, Stamatakis E et al. (2005) Physical activity patterns in nonobese and obese children assessed using minute-by-minute accelerometry. Int J Obes (Lond) 29, 1070-1076.

6. Planinsec J \& Matejek C (2004) Differences in physical activity between non-overweight, overweight and obese children. Coll Antropol 28, 747-754.

7. Newby PK (2007) Are dietary intakes and eating behaviors related to childhood obesity? A comprehensive review of the evidence. J Law Med Ethics 35, 35-60.

8. US Department of Agriculture \& US Department of Health and Human Services (2010) Dietary Guidelines for Americans, 2010. Washington, DC: US Government Printing Office.

9. Fraser LK, Edwards KL, Cade JE et al. (2011) Fast food, other food choices and body mass index in teenagers in the United Kingdom (ALSPAC): a structural equation modelling approach. Int J Obes (Lond) 35, 1325-1330.

10. Spence LA, Cifelli CJ \& Miller GD (2011) The role of dairy products in healthy weight and body composition in children and adolescents. Curr Nutr Food Sci 7, 40-49.

11. Kafatos A, Linardakis M, Bertsias G et al. (2005) Consumption of ready-to-eat cereals in relation to health and diet indicators among school adolescents in Crete, Greece. Ann Nutr Metab 49, 165-172.

12. Kelishadi R, Pour MH, Sarraf-Zadegan N et al. (2003) Obesity and associated modifiable environmental factors in Iranian adolescents: Isfahan Healthy Heart Program - Heart Health Promotion from Childhood. Pediatr Int 45, 435-442.

13. Brisbois TD, Farmer AP \& McCargar LJ (2012) Early markers of adult obesity: a review. Obes Rev 13, 347-367.

14. Instituto Nacional Estatística (2003) Estimativas da População Residente, segundo Grandes Grupos Etários e Sexo. http://www.ine.pt/ (accessed November 2008).

15. Instituto Nacional de Estatística (2011) Estimativas da População Residente, segundo Local de Residência e Sexo. http://www.ine.pt/xportal/xmain?xpid=INE\&xpgid= ine_indicadores\&indOcorrCod $=0005889 \&$ selTab $=$ tab0 (accessed May 2012). 
16. European Union (1997) União Europeia. Europa Glossário. http://europa.eu/scadplus/glossary/outermost_regions_en.htm (accessed January 2011).

17. Cole TJ, Bellizzi MC, Flegal KM et al. (2000) Establishing a standard definition for child overweight and obesity worldwide: international survey. BMJ 320, 1240-1243.

18. Cole TJ, Flegal KM, Nicholls D et al. (2007) Body mass index cut offs to define thinness in children and adolescents: international survey. BMJ 335, 194.

19. Moreno LA, Mesana MI, Gonzalez-Gross M et al. (2007) Body fat distribution reference standards in Spanish adolescents: the AVENA Study. Int J Obes (Lond) 31, 1798-1805.

20. Zimmet P, Alberti KG, Kaufman F et al. (2007) The metabolic syndrome in children and adolescents - an IDF consensus report. Pediatr Diabetes 8, 299-306.

21. Al-Hazzaa HM, Abahussain NA, Al-Sobayel HI et al. (2012) Lifestyle factors associated with overweight and obesity among Saudi adolescents. BMC Public Health 12, 354.

22. McCarthy HD \& Ashwell M (2006) A study of central fatness using waist-to-height ratios in UK children and adolescents over two decades supports the simple message - 'keep your waist circumference to less than half your height'. Int J Obes (Lond) 30, 988-992.

23. Taylor RW, Jones IE, Williams SM et al. (2000) Evaluation of waist circumference, waist-to-hip ratio, and the conicity index as screening tools for high trunk fat mass, as measured by dual-energy X-ray absorptiometry, in children aged 3-19y. Am J Clin Nutr 72, 490-495.

24. Tanner JM \& Whitehouse RH (1976) Clinical longitudinal standards for height, weight, height velocity, weight velocity, and stages of puberty. Arch Dis Child 51, 170-179.

25. Instituto Nacional de Estatística (2011) Classificação Portuguesa das Profissões 2010. Lisboa: Instituto Nacional de Estatística, IP.

26. Instituto Nacional de Estatística (1994) Classificação Nacional de Profissões. Lisboa: Instituto Nacional de Estatística, IP.

27. Lopes C, Aro A, Azevedo A et al. (2007) Intake and adipose tissue composition of fatty acids and risk of myocardial infarction in a male Portuguese community sample. J Am Diet Assoc 107, 276-286

28. Willett W (editor) (1998) Food frequency methods. In Nutritional Epidemiology, 2nd ed., pp. 74-100. New York: Oxford University Press.

29. Silva D, Rego C \& Guerra A (2004) Characterization of food habits and comparative study between two methods of food assessment in adolescents. Rev Aliment Hum 10, 33-40.

30. Telama R, Yang X, Laakso L et al. (1997) Physical activity in childhood and adolescence as predictor of physical activity in young adulthood. Am J Prev Med 13, 317-323.

31. Mota J \& Esculcas C (2002) Leisure-time physical activity behavior: structured and unstructured choices according to sex, age, and level of physical activity. Int J Behav Med $\mathbf{9}$, 111-121.

32. Raitakari OT, Porkka KV, Taimela S et al. (1994) Effects of persistent physical activity and inactivity on coronary risk factors in children and young adults. The Cardiovascular Risk in Young Finns Study. Am J Epidemiol 140, 195-205.

33. Hosmer DW \& Lemeshow S (1989) Applied Logistic Regression. New York: John Wiley \& Sons.

34. Mu Y, Edwards JR, Horan TC et al. (2011) Improving riskadjusted measures of surgical site infection for the national healthcare safety network. Infect Control Hosp Epidemiol 32, 970-986.

35. Goldberg GR, Black AE, Jebb SA et al. (1991) Critical evaluation of energy intake data using fundamental principles of energy physiology: 1. Derivation of cut-off limits to identify under-recording. Eur J Clin Nutr 45, 569-581.

36. Black AE (2000) Critical evaluation of energy intake using the Goldberg cut-off for energy intake: basal metabolic rate. A practical guide to its calculation, use and limitations. Int J Obes Relat Metab Disord 24, 1119-1130.

37. Black AE (2000) The sensitivity and specificity of the Goldberg cut-off for EI:BMR for identifying diet reports of poor validity. Eur J Clin Nutr 54, 395-404.

38. Bradlee ML, Singer MR, Qureshi MM et al. (2010) Food group intake and central obesity among children and adolescents in the Third National Health and Nutrition Examination Survey (NHANES III). Public Health Nutr 13, 797-805.

39. Hirschler V, Oestreicher K, Beccaria M et al. (2009) Inverse association between insulin resistance and frequency of milk consumption in low-income Argentinean school children. I Pediatr 154, 101-105.

40. Flegal KM \& Ogden CL (2011) Childhood obesity: are we all speaking the same language? Adv Nutr 2, 159S-166S.

41. Cowell CT, Briody J, Lloyd-Jones S et al. (1997) Fat distribution in children and adolescents - the influence of sex and hormones. Horm Res 48, Suppl. 5, 93-100.

42. Taylor RW, Jones IE, Williams SM et al. (2002) Body fat percentages measured by dual-energy X-ray absorptiometry corresponding to recently recommended body mass index cutoffs for overweight and obesity in children and adolescents aged 3-18y. Am J Clin Nutr 76, 1416-1421.

43. Moreira P, Padez C, Mourao I et al. (2005) Dietary calcium and body mass index in Portuguese children. Eur J Clin Nutr 59, 861-867.

44. Vergnaud AC, Peneau S, Chat-Yung S et al. (2008) Dairy consumption and 6-y changes in body weight and waist circumference in middle-aged French adults. $\mathrm{Am} \mathrm{J}$ Clin Nutr 88, 1248-1255.

45. Huh SY, Rifas-Shiman SL, Rich-Edwards JW et al. (2010) Prospective association between milk intake and adiposity in preschool-aged children. J Am Diet Assoc 110, 563-570.

46. Noel SE, Ness AR, Northstone K et al. (2011) Milk intakes are not associated with percent body fat in children from ages 10 to 13 years. J Nutr 141, 2035-2041.

47. Dougkas A, Reynolds CK, Givens ID et al. (2011) Associations between dairy consumption and body weight: a review of the evidence and underlying mechanisms. Nutr Res Rev (Epublication ahead of print version).

48. Morris KL \& Zemel MB (2005) 1,25-Dihydroxyvitamin $\mathrm{D}_{3}$ modulation of adipocyte glucocorticoid function. Obes Res 13, 670-677.

49. Masuzaki H, Paterson J, Shinyama H et al. (2001) A transgenic model of visceral obesity and the metabolic syndrome. Science 294, 2166-2170.

50. Kosti RI, Panagiotakos DB, Zampelas A et al. (2008) The association between consumption of breakfast cereals and BMI in schoolchildren aged 12-17 years: the VYRONAS study. Public Health Nutr 11, 1015-1021.

51. Williams BM, O'Neil CE, Keast DR et al. (2009) Are breakfast consumption patterns associated with weight status and nutrient adequacy in African-American children? Public Health Nutr 12, 489-496.

52. Albertson AM, Anderson GH, Crockett SJ et al. (2003) Ready-to-eat cereal consumption: its relationship with BMI and nutrient intake of children aged 4 to 12 years. J Am Diet Assoc 103, 1613-1619.

53. Albertson AM, Thompson D, Franko DL et al. (2009) Prospective associations among cereal intake in childhood and adiposity, lipid levels, and physical activity during late adolescence. J Am Diet Assoc 109, 1775-1780.

54. Barton BA, Eldridge AL, Thompson D et al. (2005) The relationship of breakfast and cereal consumption to nutrient intake and body mass index: the National Heart, Lung, and Blood Institute Growth and Health Study. J Am Diet Assoc 105, 1383-1389.

55. Hardy R, Wadsworth M \& Kuh D (2000) The influence of childhood weight and socioeconomic status on change in 
adult body mass index in a British national birth cohort. Int J Obes Relat Metab Disord 24, 725-734.

56. Lakshman R, Zhang J, Koch FS et al. (2013) Higher maternal education is associated with favourable growth of young children in different countries. J Epidemiol Community Health 67, 595-602.

57. Koupil I \& Toivanen P (2008) Social and early-life determinants of overweight and obesity in 18-year-old Swedish men. Int J Obes (Lond) 32, 73-81

58. Kim Y \& Lee S (2009) Physical activity and abdominal obesity in youth. Appl Physiol Nutr Metab 34, 571-581.

59. Klein-Platat C, Oujaa M, Wagner A et al. (2005) Physical activity is inversely related to waist circumference in 12 -y-old French adolescents. Int J Obes (Lond) 29, 9-14.

60. Ortega FB, Ruiz JR \& Sjostrom M (2007) Physical activity, overweight and central adiposity in Swedish children and adolescents: the European Youth Heart Study. Int J Behav Nutr Phys Act 4, 61.

61. Matthews VL, Wien M \& Sabate J (2011) The risk of child and adolescent overweight is related to types of food consumed. Nutr J 10, 71.

62. Tohill BC, Seymour J, Serdula M et al. (2004) What epidemiologic studies tell us about the relationship between fruit and vegetable consumption and body weight. Nutr Rev 62, 365-374.

63. Bandini LG, Schoeller DA, Cyr HN et al. (1990) Validity of reported energy intake in obese and nonobese adolescents. Am J Clin Nutr 52, 421-425.
64. Araujo J, Severo M, Lopes C et al. (2011) Food sources of nutrients among 13-year-old Portuguese adolescents. Public Health Nutr 14, 1970-1978.

65. Collins CE, Watson J \& Burrows T (2010) Measuring dietary intake in children and adolescents in the context of overweight and obesity. Int J Obes (Lond) 34, 1103-1115.

66. Sweeting H \& West P (2002) Gender differences in weight related concerns in early to late adolescence. $J$ Epidemiol Community Health 56, 700-701.

67. Maynard LM, Wisemandle W, Roche AF et al. (2001) Childhood body composition in relation to body mass index. Pediatrics 107, 344-350.

68. Bacha F, Saad R, Gungor N et al. (2003) Obesity, regional fat distribution, and syndrome $\mathrm{X}$ in obese black versus white adolescents: race differential in diabetogenic and atherogenic risk factors. J Clin Endocrinol Metab 88, $2534-2540$.

69. Wajchenberg BL (2000) Subcutaneous and visceral adipose tissue: their relation to the metabolic syndrome. Endocr Rev 21, 697-738.

70. Savva SC, Tornaritis M, Savva ME et al. (2000) Waist circumference and waist-to-height ratio are better predictors of cardiovascular disease risk factors in children than body mass index. Int $J$ Obes Relat Metab Disord 24, 1453-1458.

71. Hara M, Saitou E, Iwata F et al. (2002) Waist-to-height ratio is the best predictor of cardiovascular disease risk factors in Japanese schoolchildren. J Atheroscler Thromb 9, 127-132. 